\title{
Por sus basuras los reconoceréis: La identificación arqueozoológica de una comunidad dimmí cristiana en la Qurțuba tardoandalusí (Cercadilla, siglo XII)
}

\author{
By their rubbish you will recognise them: The zooarchaeological identification \\ of a dhimmi christian community in late andalusi Qurtuba (Cercadilla, $12^{\text {th }}$ \\ century)
}

\author{
Marcos García García ${ }^{1}$ \\ Marta Moreno García \\ $M^{a}$ del Camino Fuertes Santos ${ }^{3}$ \\ Rafael Hidalgo Prieto ${ }^{4}$
}

Recibido: 5/10/2020

Aprobado: $11 / 02 / 2021$

Publicado: 10/05/2021

\section{RESUMEN}

Se presentan los resultados del estudio arqueozooló gico del material recuperado de una unidad de poblamiento localizada en la Zona Arqueológica de Cercadilla, en el sector periurbano de madinat Qurtuba, frecuentada durante el s. XII. Los datos que se analizan se derivan, de un lado, de un conjunto faunístico identificado como residuos de alimentación y, de otro lado, de una valva derecha perforada de vieira procedente de un nivel de frecuentación. La elevada frecuencia de restos de porcino refleja un comportamiento alimentario que transgrede la principal norma dietética de la ortopraxis islámica; mientras que la valva fue identificada como una concha de peregrino vinculada al universo simbólico del mundo cristiano medieval. Tomados conjuntamente, y en coherencia con la historia ocupacional de Cercadilla, ambos resultados permiten sugerir la adscripción sociocultural o etnorreligiosa cristiana de al menos una parte del grupo que residió en este espacio, enriqueciendo y complejizando nuestra concepción del panorama social tardoandalusí.

Palabras clave: arqueozoología, mozárabes, alAndalus, alimentación, identidad.

\section{SUMMARY}

This article presents the results of the zooarchaeological analysis of the faunal material recovered from a domestic and agro-industrial unit located in the Archaeological Area of Cercadilla, in the outskirts of madinah Qurtuba, that was inhabited during the 12th century. The data source is twofold: on the one hand, a sample of animal bones from a closed context identified as food refuse; on the other, a pierced right scallop shell from a living floor. The high frequency of pig remains indicates the consumption of pork, a behavior that implies a transgression of the main dietary rule of the Islamic orthopraxis, whereas the scallop was identified as a pilgrim shell linked to the symbolic sphere of the medieval Christian world. Both results, along with the occupational history of Cercadilla, allow to suggest the Christian identity of this community, enriching our conception of the social landscape during the late Andalusi period.

Keywords: zooarchaeology, mozarabs, al-Andalus, food consumption, identity.

1. Postdoctoral Research Fellow. BioArCh, Department of Archaeology (University of York). C/Abelia, 11 (dcha.), 18198 Huétor Vega (Granada).marcos.garcia2@york.ac.uk. ORCID iD: https://orcid.org/0000-0002-2080-5098.

2. Científica titular. Instituto de Historia, G.I. Paleoeconomía y subsistencia de las sociedades preindustriales (Consejo Superior de Investigaciones Científicas). C/Albasanz, 26-28, 28037 (Madrid). marta.moreno@cchs.csic.es. ORCID iD: https://orcid. org/0000-0002-6735-9355

3. Coordinadora Enclaves RECA Córdoba. Grupo PAI HUM-882. Agencia Andaluza de Instituciones Culturales (AAllCC), Junta de Andalucía. Estadio Olímpicos/n, 41075 (Sevilla). camino.fuertes@juntadeandalucia.es. ORCID iD: https://orcid.org/0000-0001-9579-3143.

4. Profesor titular de universidad. Área de Arqueología (Universidad Pablo de Olavide). Ctra. de Utrera, Km. 1, 41013, (Sevilla).rhidpri@ upo.es. ORCID iD: https://orcid.org/0000-0003-3835-0711.

Cómo citar: García García, M., Moreno García, M., Fuertes Santos, M. y Hidalgo Prieto, R. (2021): Por sus basuras los reconoceréis: La identificación arqueozoo lógica de una comunidad dimmí cristiana en la Qurțuba tardoandalusí (Cercadilla, siglo XII). Arqueología Y Territorio Medieval, 28. e5797. https://doi.org/10.17561/aytm.v28.5797 


\section{INTRODUCCIÓN}

Las relaciones que los seres humanos han establecido a lo largo de la historia con los animales contienen claves esenciales para entender procesos sociales. Ello se debe a que determinadas especies faunísticas han representado históricamente -y representan en la actualidad - mucho más que simples recursos de los que los humanos nos servimos con el fin de satisfacer necesidades subsistenciales básicas, desempeñando igualmente funciones sociales como resultado de los atributos culturales que se derivan de la posesión, la gestión y el consumo de los mismos o de sus productos derivados (O'Connor, 1992).

La arqueozoología, consistente en el estudio de restos faunísticos recuperados mediante metodología arqueológica, presenta en este sentido un potencial informativo de primer orden que permite no solo conocer qué animales constituyeron la base alimenticia de las sociedades pretéritas, sino también arrojar luz sobre la posible identidad de los grupos humanos responsables de la acumulación de los residuos examinados. Así, mediante el reconocimiento de los hábitos de consumo, esta disciplina de análisis arqueológico permite adscribir los actores sociales del pasado a grupos étnicos (sensu BARTH, 1969) específicos.

El caso de al-Andalus representa, al hilo de estas consideraciones, un laboratorio de especial interés para la aplicación de esta línea de investigación, habida cuenta de que la fábrica social de dicha entidad histórica estuvo compuesta por diversas comunidades socioculturales o etnorreligiosas cuya presencia no siempre aparece bien reflejada en otros tipos de registro, particularmente el escrito. En concreto, como trataremos de poner de manifiesto a lo largo de las siguientes páginas, el análisis arqueozoológico posibilita la generación de conocimiento relativo al grado de adecuación de los patrones alimentarios de los actores sociales a la normativa dietética propia del orden islamizado y, de esta forma, reconocer el grado de islamización sociocultural de los grupos cuyo consumo se refleja en el registro arqueofaunístico.

En este trabajo nos proponemos presentar algunos de los resultados obtenidos a lo largo de un proyecto de investigación más amplio que trató de explorar, desde un enfoque histórico y arqueozoológico, las formas de interacción entre humanos y animales durante la Alta Edad Media en el sudeste peninsular ${ }^{5}$. La atención se centrará sobre uno de los casos de estudio considerados en aquella ocasión, derivado del análisis arqueofaunístico del material recuperado de una unidad de poblamiento periurbano que se desarrolló durante el s. XII en la Zona Arqueológica de Cercadilla (Córdoba). Los datos que serán presentados y discutidos permiten sugerir como hipótesis de trabajo la identidad cristiana de, al menos, parte de la comunidad que frecuentó dicha unidad de poblamiento. Así pues, el interés fundamental de nuestra aportación radica en la presentación de evidencia arqueológica que revela la posible pervivencia de un grupo dimmí cristiano (o mozárabe) en la Qurtuba del s. XII, momento en el que comúnmente la historiografía ha venido situando el final de dichas comunidades en al-Andalus como resultado de la persecución, deportación, emigración o conversión (AILLET, 2010, 2011; CARMONA, 2007; FIERRO, 1997; GARCÍA SANJUÁN, 2004). Por este motivo, el presente trabajo aspira a demostrar el potencial informativo de la disciplina arqueozoológica para identificar la existencia de formas sociales y culturales cuya presencia aparece opacada en las fuentes documentales, contribuyendo de esta forma a generar una imagen más compleja y rica del panorama sociocultural tardoandalusí.

Para ello, estimamos necesario introducir en primer lugar una reflexión de orden teórico en torno a dos cuestiones como son, de un lado, el uso de los patrones de alimentación como marcador diagnóstico para estudiar la identidad y, de otro lado, su aplicabilidad en

5. Dicha investigación conforma la tesis doctoral del primer autor defendida en 2019 en la Universidad de Granada (GARCÍA GARCíA, 2019), elaborada gracias a una ayuda predoctoral del programa FPU (Formación de Profesorado Universitario) (Ref. AP2012-4717) financiada por el Ministerio de Educación, Cultura y Deporte 
el caso específico de una sociedad islámica medieval como fue al-Andalus. En segundo lugar, tras la presentación del caso de estudio de Cercadilla se exponen los resultados del análisis arqueozoológico antes de pasar a la discusión de la evidencia arqueofaunística y a la interpretación en términos históricos de las implicaciones económicas y socioculturales que de ellos se desprenden.

\section{ALIMENTACIÓN E IDENTIDAD: PERSPECTIVASARQUEOZOOLÓGICAS}

El papel crucial que las prácticas dietéticas desempeñan en la conformación de la sociedad y en la definición, en sus múltiples dimensiones, de la identidad de los seres humanos es un fenómeno bien conocido que presenta un particular potencial analítico en el marco de este trabajo. La alimentación representa un ámbito de la experiencia humana enormemente amplio y complejo en el que se imbrican de manera orgánica aspectos de naturaleza económica, ecológica, social, cultural, simbólica y religiosa. Se trata, así pues, de un «acto social altamente condensado» (APPADURAI, 1981) cuyo estudio permite acceder al conocimiento de las sociedades a diferentes niveles de análisis. De entre todos los aspectos relacionados con la esfera de la alimentación, fundamentalmente nos interesa incidir sobre el papel decisivo que el alimento y su consumo desempeña en la construcción y el reforzamiento de los vínculos sociales, actuando como la argamasa que forma los lazos familiares y sociales mientras crea, al mismo tiempo, al individuo (ATALAY y HASTORF, 2006). Además, como acto social producido y reproducido diariamente mediante patrones repetitivos y recurrentes (ROZIN, 1987: 196), ocupa un lugar fundamental entre las prácticas que sustentan la vida cotidiana de los seres humanos organizados socialmente y que sirven para inculcar habitus, esto es, el conjunto de disposiciones incorporadas que estructuran la acción de los individuos y que, de manera inconsciente, construyen percepciones de identidad y diferencia (BOURDIEU, 1980). Por todo lo anterior, la alimentación juega un papel activo en la creación y el mantenimiento de los procesos sociales y culturales, representando un medio particularmente apropiado para aprehender las formas a partir de las cuales las sociedades y las culturas se constituyen y se transforman a lo largo del tiempo (SHERRATT, 1995).

De manera más específica, para nuestros objetivos actuales resulta necesario incidir sobre la relevancia de la esfera alimentaria en relación a la formación y el mantenimiento de la identidad, un concepto complejo por su naturaleza poliédrica y fluida que, siguiendo a J. C. TURNER (1999), puede definirse como la identificación propia y personal de los individuos en términos de pertenencia grupal con las normas, los ideales y los comportamientos de dichos grupos. Las identidades, por lo tanto, se construyen a través de las diferencias establecidas con otros individuos y/u otras comunidades (BARTH, 1969; HALL, 1996), diferencias que en gran medida tienden a vehicularse a través de comportamientos, experiencias y prácticas cotidianas que expresan y reproducen sentimientos de pertenencia étnica o comunal (BENTLEY, 1987) y que, en consecuencia, presentan una expresión material arqueológicamente reconocible (INSOLL, 2007; JONES, 1997; LUCY, 2005).

En tal sentido, conviene señalar que entre los diversos mecanismos a través de los cuales los grupos humanos establecen y conservan las «fronteras culturales» que los sitúan en referencia a otros grupos o comunidades (BARTH, 1969), los alimentos y la forma de prepararlos para su consumo representan uno de los factores que de manera más directa enfatizan la similitud o la diferencia comunal, resultando por ello fundamentales en la demarcación de afinidades y alteridades identitarias sociales y culturales (FISCHLER, 1988; LUCY, 2005).

De todos los aspectos relacionados con el plano de la alimentación que de manera más evidente formalizan esta realidad destacan los tabúes alimentarios dado que, mediante la interdicción sobre el consumo de ciertos alimentos, son empleados como recursos metafóricos que sirven para posicionar en el mundo tanto los alimentos como a las personas (FISCHLER, 1988), incluso cuando estas no son conscientes 
de ello (SCHOLLIERS, 2001). En palabras de M. DOUGLAS (1984: 9), los tabúes alimentarios «materializan las abstracciones espirituales de los seres humanos» y, de esta forma, suponen la traslación al campo de la práctica de la adscripción identitaria de los individuos a los grupos a los que pertenecen (BENKHEIRA, 1997). Así, al proscribir el consumo de alimentos específicos (fundamentalmente cárnicos [FIDDES, 1991; SIMOONS, 1994]) a un grupo humano concreto, los tabúes resultan particularmente apropiados para su estudio arqueozoológico al representar una vía de acceso al conocimiento de los hábitos de consumo regulares y mantenidos a lo largo del tiempo de los productos alimentarios de origen animal por parte de las poblaciones del pasado. Por lo tanto, mediante el examen de la frecuencia con que aparecen representadas en el registro arqueofaunístico ciertas especies animales en contextos históricos específicos, como el investigado en este trabajo, la arqueozoología nos faculta para caracterizar el contexto social en que se realizó el consumo y, de esta forma, adscribir culturalmente a los grupos responsables de la generación de los residuos objeto de análisis.

\section{INDICADORES ARQUEOZOOLÓGICOS PARA EL ESTUDIO DE LA IDENTIDAD EN AL-ANDALUS}

En el marco de la investigación que dio origen al estudio del caso que nos ocupa, empleamos como herramienta analítica la noción de islamización social para referirnos al proceso de transformación de la antigua Hispania que conllevó la implantación del orden social islámico (GARCÍA GARCÍA, 2019). Desde nuestro punto de vista, al-Andalus fue, antes que nada, una sociedad islamizada, un calificativo equivalente al sentido que HODGSON (1974: 59) otorga al término «islamicate» para designar al complejo social y cultural históricamente asociado con el islam y los musulmanes, y que afecta tanto a los mismos musulmanes como a la población no musulmana.

Nuestra concepción del proceso referido parte, siguiendo a autores como T. INSOLL
(1999) y T. ASAD (1986), de una consideración del islam como un principio o tradición estructural integrada tanto por un sistema de creencias o fundamentos de fe (una ortodoxia), como por un marco de prácticas y disposiciones sociales que conforman el marco de acción y de expresión cultural del islam (una ortopraxis). Desde este enfoque, estimamos que la dimensión religiosa del islam se manifiesta en el plano terrenal a través de disposiciones sociales que son tanto de naturaleza metafísica como material, y que juntas conforman la identidad islámica de los actores sociales. Nuestro interés, por lo tanto, no pasa tanto por conocer en qué creyeron las personas en el pasado, sino por evaluar qué hicieron esas personas como consecuencia de sus creencias, algo que sí podemos observar a través del estudio de la expresión material de ciertos comportamientos en el registro arqueológico (BENDER et al., 1997: 148; INSKIP, 2013: 18; INSOLL, 2004: 47).

En tal sentido, y engarzando con las reflexiones previas en torno al potencial de la arqueozoología para el estudio de la identidad-particularmente a través de la frecuencia que presentan en el registro arqueofaunístico ciertas especies animales sobre las que recae un tabú-, cabe introducir en la discusión el estrecho vínculo existente entre prácticas alimentarias e identidad en el mundo islamizado medieval en general, y en al-Andalus en particular.

Entre los diversos ámbitos de la experiencia humana que aparecen claramente regulados y normativizados en la doctrina básica del islam se encuentran una serie de disposiciones que afectan directamente al plano de la alimentación (BENKHEIRA, 1997, 2000; COOK, 1986; FAROUK et al., 2015). Las prescripciones a este respecto son numerosas y atañen a aspectos muy diversos que, en el caso concreto de los alimentos de origen animal, incluyen desde qué productos son lícitos para su consumo y cuáles ilícitos, hasta cómo deben ser sacrificados los animales de los que se derivan dichos productos. En el caso específico de al-Andalus, los juristas mālikíes elaboraron 
y establecieron diversas normas basadas tanto en el Corán como en la sunna que los creyentes debían contemplar en su alimentación y que conocemos gracias a trabajos de enorme interés como los de $M^{a}$. ARCAS (1996), A. GARCÍA SANJUÁN (2002), A. FERNÁNDEZ (2003) o J. SAFRAN (2003), en donde la cuestión que se refiere a la legalidad del consumo por parte de los musulmanes de los alimentos de los dimmíes (cristianos y judíos protegidos) ocupa un lugar central.

En el marco de esta contribución nos interesa específicamente una de las prescripciones alimentarias que, por su carácter esencial, no fue objeto de ningún tipo de controversia como parte de estas ni de ninguna otra de las interpretaciones, coetáneas o no, del texto coránico ni de la tradición profética (BENKHEIRA, 2000, 2002; KRUK, 2019). Evidentemente, nos referimos a uno de los pocos alimentos considerado de naturaleza impura (naŷāsa) por todas las doctrinas jurídicas del islam -incluyendo la mālikí, «la "espina dorsal" de la identidad de al-Andalus» (FIERRO, 2004: 137)- como es el porcino (janzirr), sobre el cual recae la prohibición absoluta (harām) de su consumo. Aunque ni en el Corán ni en ninguna de las interpretaciones posteriores de época medieval encontramos una distinción específica entre cerdo doméstico y jabalí salvaje (BENKHEIRA, 2002; VIRÉ, 1986) asumimos que la prohibición incluye igualmente al «doublet sauvage du porc domestique» (BENKHEIRA, 1995: 85), por lo que resulta apropiado el empleo de la categoría taxonómica de «suidos» para referirnos a estos animales, puesto que incluye a ambas especies.

A pesar de que el origen del tabú islámico del consumo de suidos ha sido y continúa siendo objeto de controversia y debate historiográfico, lo que está fuera de toda duda es que esta interdicción aparece recogida desde las primeras versiones conocidas del texto coránico (BENKHEIRA, 2000: 51), considerado como la pura palabra divina revelada a la humanidad a través del Profeta y, como tal, eterna e inalterable (MARíN, 2004). Del mismo modo, y al margen de esta fuente esencial de doctrina, disponemos de abundantes referencias escritas de naturaleza no estrictamente religiosa que reflejan tanto la antigüedad de este precepto fundamental de la normativa dietética islámica como lo arraigado de su observancia como parte del acervo identitario arabo-islámico. Así se desprende, por ejemplo, de varias de las masāil de la 'Utbiyya, la compilación de audiciones del alfaquí al-'Utbī (m. 869) estudiada por A. FERNÁNDEZ (2003); del documento conocido como Šurūt 'Umar, que contiene las normas a las que los no musulmanes deben someterse en las tierras conquistadas (LEVY-RUBIN, 2011, 2013); del recuento de las conquistas en Siria del califa 'Abd al-Malik (m. 705), que ordenó el abatimiento de todos los cerdos de la región (BENKHEIRA, 2002: 46); de las opiniones de diversos juristas mālikíes vertidas en los tratados de fiah desde el s. VIII al XVI examinados por Mª. ARCAS (2016: 96), en las que queda patente «la prohibición absoluta y unánime de la carne de cerdo» o, finalmente, del tratado de dietética de Ibn Zuhr que excluye la mención a las propiedades nutricionales de la carne de este animal debido a que «la ley islámica prohíbe que se cite» (GARCÍA SÁNCHEZ, 1992: 59).

Así pues, si concebimos la islamización social como el proceso mediante el cual los comportamientos propios de la ortopraxis del islam medieval pasaron a formar parte de la fábrica social y cultural de la nueva sociedad andalusí, estimamos que la adopción de la normativa dietética islámica -y, en particular, el abandono del consumo de porcino- fue uno de los principales elementos empleados desde el periodo de emergencia de al-Andalus en el terreno de la cotidianidad para demarcar la pertenencia de los individuos a la comunidad musulmana.

Este triple nexo existente entre alimentación, ortopraxis e identidad islámica resulta de particular relevancia para nuestros intereses. Dado que la prohibición del consumo de suidos constituye el símbolo más elemental de pertenencia al islam y, al mismo tiempo, uno de sus modos de actualización (BENKHEIRA, 1997, 1999), el empleo como rasgo diagnóstico de la 
observancia (o inobservancia) de esta prescripción dietética central en la ortopraxis del islam medieval nos faculta para arrojar luz sobre la identidad de los agentes consumidores y, de esta forma, reconocer el grado de inserción de los mismos en el orden social islamizado. La evidencia arqueozoológica disponible hasta la fecha para el conjunto de al-Andalus examinada conjuntamente como parte de la investigación matriz en que se inserta el presente estudio (GARCÍA GARCÍA, 2019) así lo atestigua, representando un refrendo arqueológico al carácter diagnóstico que presenta la frecuencia de suidos en el registro arqueofaunístico para examinar esta y otras cuestiones.

Con estos mimbres, a lo largo de las siguientes páginas trataremos de aplicar el enfoque de análisis aquí esbozado a un caso de estudio específico, tratando de demostrar el potencial informativo de la disciplina arqueozoológica para conocer la posible adscripción sociocultural del grupo humano residente durante el s. XII en la Zona Arqueológica de Cercadilla (Córdoba).

\section{LA ZONA ARQUEOLÓGICA DE CERCA- DILLA (CÓRDOBA)}

Cercadilla se localiza al noroeste del recinto amurallado de madinat Qurtuba, la actual ciudad de Córdoba (Figs. 1a y b). Durante las obras de soterramiento de la estación de ferrocarril en 1991 aparecieron los restos de un complejo monumental de época romana que, tras ser objeto de uno de los casos más ominosos de destrucción de patrimonio arqueológico en nuestro país (Figs. 1c y d), fueron intervenidos a lo largo de varias campañas de excavaciones arqueológicas de urgencia que permitieron evaluar los daños producidos y ofrecer una imagen nítida de aquello que las palas excavadoras no llegaron a destruir. A pesar de la gravedad de las afecciones a los contextos arqueológicos durante la destrucción de la primavera de 1991 y el consiguiente levantamiento de las nuevas estaciones de ferrocarril y autobuses, la investigación llevada a cabo ha permitido comprender la configuración formal de un complejo arquitectónico de época romana, así como la secuencia histórica posterior durante la Tardoantigüedad y la Edad Media (Fig. 1e). Si bien es mucho lo que se ha perdido para el conocimiento de este espacio, a partir de la labor arqueológica llevada a cabo desde el inicio de las excavaciones ha sido posible documentar una secuencia de ocupación de enorme interés para la comprensión de la transformación de una sociedad urbana tardoantigua en andalusí.

Aunque nuestro interés se centra exclusivamente sobre la última fase de ocupación medieval de este espacio acaecida durante el s. XII, resulta preciso introducir, si quiera de manera sintética, los precedentes históricos del asentamiento dado que, como veremos, son relevantes para la interpretación de los resultados obtenidos en este trabajo.

\subsection{Secuencia general de ocupación: de la época romana a la fitna del s. XI}

El principal elemento estructurador del paisaje de este sector cordobés durante época preandalusí se define a partir de la construcción, entre finales del s. III e inicios del IV, de un conjunto edilicio de vastas dimensiones identificado como palacio y sede del emperador Maximiano Hercúleo (HIDALGO, 1996, 2004, 2014, 2016; HIDALGO y VENTURA, 1994). Tras el abandono de sus funciones originales en época tardorromana, la evidencia arqueológica permite constatar la transformación parcial, al menos desde el s. VI, de este complejo en un centro de culto cristiano conformado tanto por un conjunto de edificios del palacio romano, que constituirían su núcleo principal, como por una extensa necrópolis que mantuvo una continuidad funcional, topográfica y ritual -si bien tras una reducción de su superficie durante el s. X- hasta principios del s. XI (FUERTES, 2018; FUERTES e HIDALGO, 2010; FUERTES et al., 2007; HIDALGO, 2002, 2016; ORTIZ 2003). De manera sincrónica a la vida de este complejo cristiano disponemos igualmente de evidencias que indican el desarrollo de una intensa ocupación de carácter doméstico y residencial durante época emiral 


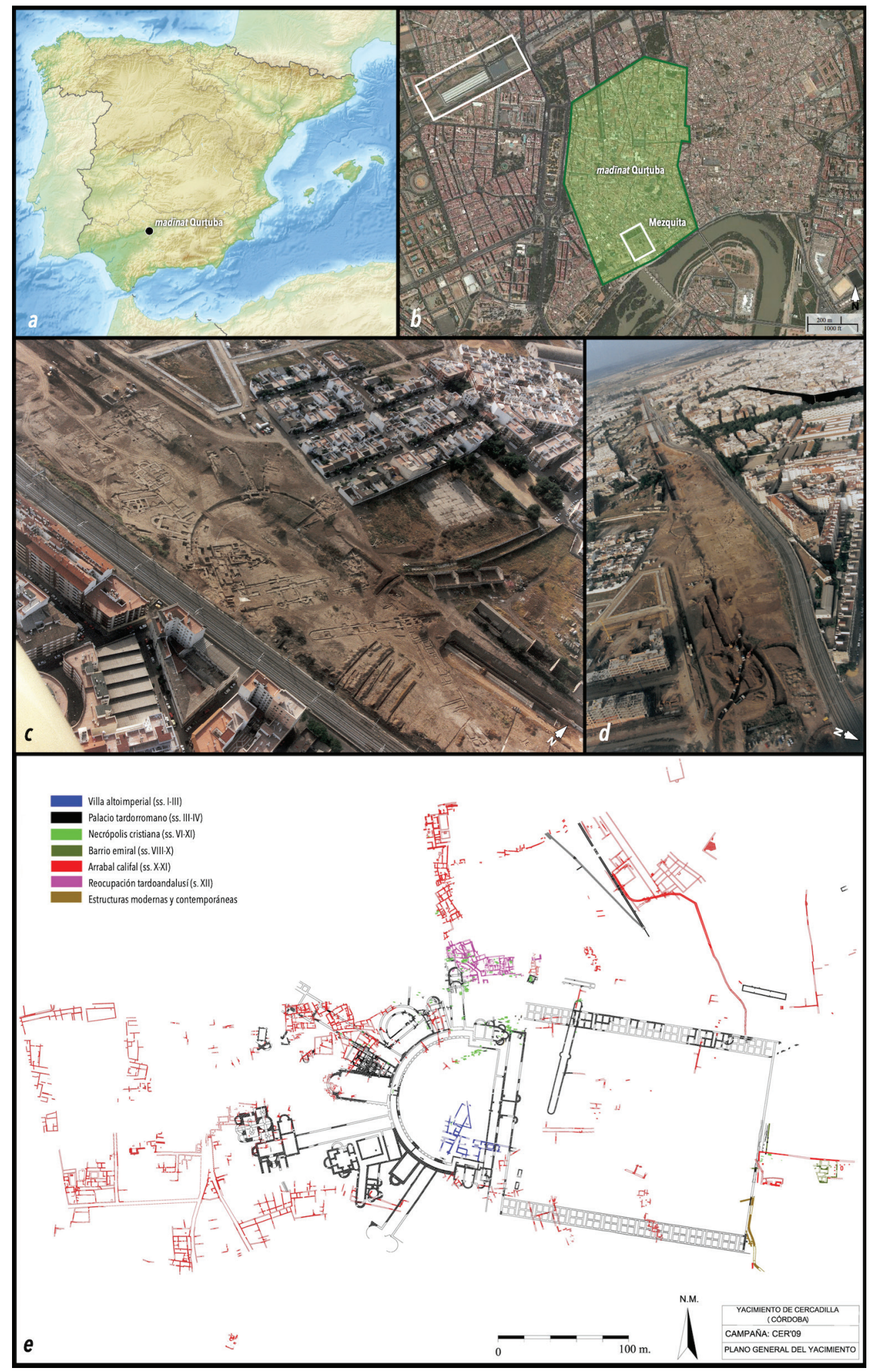

Fig. 1. Zona Arqueológica de Cercadilla: a) Localización en el contexto peninsular; b) Situación de Cercadilla (rectángulo blanco) respecto al núcleo urbano de madinnat Qurțuba; c-d) Vistas aéreas del espacio afectado por las obras (playa de vías) de la estación de ferrocarril en 1992 (R. Hidalgo); e) Planimetría del yacimiento y fases de ocupación ( $M^{a}$. del C. Fuertes y R. Hidalgo). 
(ss. VIII-pr. s. X) que, posteriormente, adquiriría un carácter plenamente urbano en época califal mediante su integración como arrabal en la enorme conurbación que desde mediados del s. X se desarrolló en el espacio extramuros de la capital de al-Andalus (ACIÉN y VALLEJO, 1998; LEÓN y MURILLO, 2014). El convulso periodo de la fitna de inicios del s. XI implicó el repliegue de la población al interior de la madina amurallada (BLANCO, 2014; LEÓN y BLANCO, 2010), quedando tanto el espacio habitado de Cercadilla como la necrópolis cristiana asociada abandonados hasta el s. XII, cuando se produjo una nueva fase de reocupación de la que se deriva el material aquí examinado y de la que nos ocuparemos en detalle a continuación.

Antes de ello, es necesario detenerse sobre una cuestión de trascendental importancia al hilo de esta síntesis de la evolución del poblamiento de Cercadilla, como es la posible identidad sociocultural y religiosa de la población asentada en este espacio durante época emiral y califal (siglos VIII-XI). Como ya se ha señalado, la presencia de un centro de culto cristiano en uso desde época tardoantigua condujo desde muy temprano a sugerir la posible filiación dimmí del grupo poblacional aquí residente, aspecto este sobre el que el análisis arqueozoológico de los hábitos de consumo de los productos animales permitió arrojar alguna luz (GARCÍA GARCÍA, 2019). A partir de dicho estudio se evidenció un elocuente patrón diacrónico consistente en la existencia de lo que pensamos razonable definir como un contexto social de consumo no islamizado en época emiral ${ }^{6}$-tal y como sugiere la abundante frecuencia de restos de suidos atribuidos a porcino doméstico y de valvas de ostra Ostrea edulis-, y un contexto islamizado durante la fase califal, reflejado en la desaparición de restos de porcino que fue interpretada como consecuencia del abandono de la cría local y, en consecuencia, del consumo de este animal.
Sin embargo, esta información no permite inferir si la población residente en Cercadilla durante época califal confesó o no la fe musulmana -y, por lo tanto, lo que se produjo fue su conversión religiosa al islam durante el s. X-, o si a lo que asistimos es a un relevo en el uso residencial de este sector de la Qurtuba andalusí entre las fases de ocupación emiral y califal por parte de dos poblaciones diferentes. Lo que los datos arqueozoológicos constatan es la identificación de contextos de consumo alimentario de productos de origen animal que, en el marco histórico en que nos movemos, consideramos posible vincular con el grado de islamización social de los agentes consumidores, entendiendo este proceso, en la línea de lo ya apuntado, como la adopción de los hábitos cotidianos propios de la ortopraxis del islam medieval en los que la abstinencia del consumo de carne de porcino desempeña un papel central.

En cualquiera de los casos, nos interesa enfatizar que la evidencia arqueológica disponible sugiere que, hasta el abandono a inicios del s. XI del arrabal y del centro de culto cristiano con necrópolis asociada, al menos un sector de la población residente en el espacio de Cercadilla se enterró siguiendo el rito cristiano a pesar de que sus hábitos de alimentación pudieran definirse en términos sociales como plenamente islamizados.

\subsection{La reocupación de Cercadilla en el siglo XII}

La fitna de principios del s. XI supuso el abandono tanto del arrabal califal de Cercadilla como del edificio de culto cristiano y la necrópolis a él asociada en uso desde el S. VI. Sin embargo, la labor arqueológica ha permitido documentar una última fase de ocupación medieval de carácter agroindustrial y doméstico durante el s. XII -un periodo que denominaremos «tardoandalusí» siguiendo

\footnotetext{
6. Contrastan estos resultados con los derivados del caso coetáneo (época emiral, med. S. VIII-pr. S. IX) del arrabal cordobés de Šaqunda, en donde destaca la ausencia total de restos de suidos entre los residuos de alimentación recuperados tanto de la Zona A (MARTÍNEZ, 2017) como de la Zona C (GARCÍA GARCÍA, 2019).
} 
las consideraciones tanto de R. BLANCO (2014: 16-17) como de E. SALINAS (2012: 2-3)- al norte $y$ este de uno de los cinco edificios absidiados del complejo romano que aún en ese momento permanecía en pie (Figs. 2a y b). Dado que el material objeto de estudio del presente trabajo se deriva de los niveles asociados a esta unidad de poblamiento, atenderemos en mayor detalle del dedicado a las fases anteriores del asentamiento a las características históricas y arqueológicas que definen la ocupación de Cercadilla durante este periodo.

La construcción de este complejo arquitectónico en el s. XII se produjo en uno de los pocos sectores libres de viviendas del por entonces abandonado arrabal califal. Por él se extendía parte de la necrópolis cristiana que había sido respetada por la reordenación urbanística del s. X, lo que explica la inexistencia de edificaciones de carácter doméstico de época emiral y califal en este sector (FUERTES, 2006). El hecho de que algunas de estas construcciones obliteren directamente un buen número de estructuras funerarias sugiere una ruptura con respecto a la dinámica de asentamiento previa. En ese mismo sentido apunta la situación general del poblamiento de este sector de Qurtuba ya que, como se ha demostrado, tras el colapso del califato omeya, el urbanismo de este espacio parece difuminarse, dejando paso a lo largo del s. XII a una reocupación selectiva de los antiguos espacios domésticos califales (BLANCO, 2014). En resumen, nos encontramos ante una ocupación de carácter relativamente aislado y periférico con respecto a la madīna que en ningún caso se desarrolló en el sentido urbanístico de la etapa precedente, ni tuvo la entidad del poblamiento de época califal (Fig. 2c). Estos rasgos definitorios de la naturaleza del poblamiento de Cercadilla durante este periodo resultan determinantes para explicar algunos de nuestros resultados.

El carácter ruinoso de las estructuras erigidas durante esta fase de ocupación, unido al arrasamiento posterior de las mismas, dificultó significativamente la definición de los espacios y la interpretación de su funcionalidad, a pesar de lo cual fue posible identificar un entramado edilicio conformado por varios módulos anejos separados por un espacio de tránsito. No se trata, por lo tanto, de una unidad estructural única, sino de un complejo arquitectónico constituido por diferentes ambientes, llegándose a diferenciar hasta doce edificios distintos que tendrían funciones tanto productivas como domésticas (FUERTES, 2006). Así parece indicar, por ejemplo, la presencia de un horno cerámico, varias piletas de decantación, además de varias estancias destinadas al almacenamiento de tinajas, en el interior de las cuales se conservaban restos de un residuo de apariencia grasienta que permitió interpretar al menos un sector de este complejo como una instalación destinada a la producción oleícola (FUERTES e HIDALGO, 2005).

El elenco cerámico recuperado de los niveles asociados a esta unidad de poblamiento no parece dejar lugar a demasiadas dudas acerca del momento en que fue frecuentada. Particularmente destaca la aparición de nuevos tipos de formas cerámicas ausentes en los niveles tanto emirales como califales como son (a) las ollas con carena en los hombros y cuellos estrangulados, o de paredes finas, cuerpo globular y cuello cilíndrico; (b) los jarros carenados con pie anular o con una inflexión pronunciada en las paredes; (c) las jarritas de cuerpo achatado, cuellos cilíndricos y pie anular; (d) los ataifores carenados y con las paredes muy exvasadas; o (e) las cazuelas vidriadas al interior (FUERTES, 2010: 197-8) (Fig. 2d). Del mismo modo, por vez primera se documenta la aplicación de la técnica del vedrío para recubrir el interior de las ollas «Tipo 8» (ibid.), marcando así un cambio significativo con respecto a la tradición anterior, ya que durante la fase califal no se observa la aplicación del vidriado en los recipientes de cocina (FUERTES, 2009, 2010). En definitiva, las características del conjunto cerámico aquí resumidas permiten vincularlo a un único horizonte cronotipológico que remite de manera directa al universo morfológico, técnico y decorativo de las producciones que se desarrollan durante época tardoandalusí en el sector intramuros de Qurtuba -el comúnmente denominado «almohade» (FUERTES, 2010; SALINAS, 2012), por lo que la dinámica de 


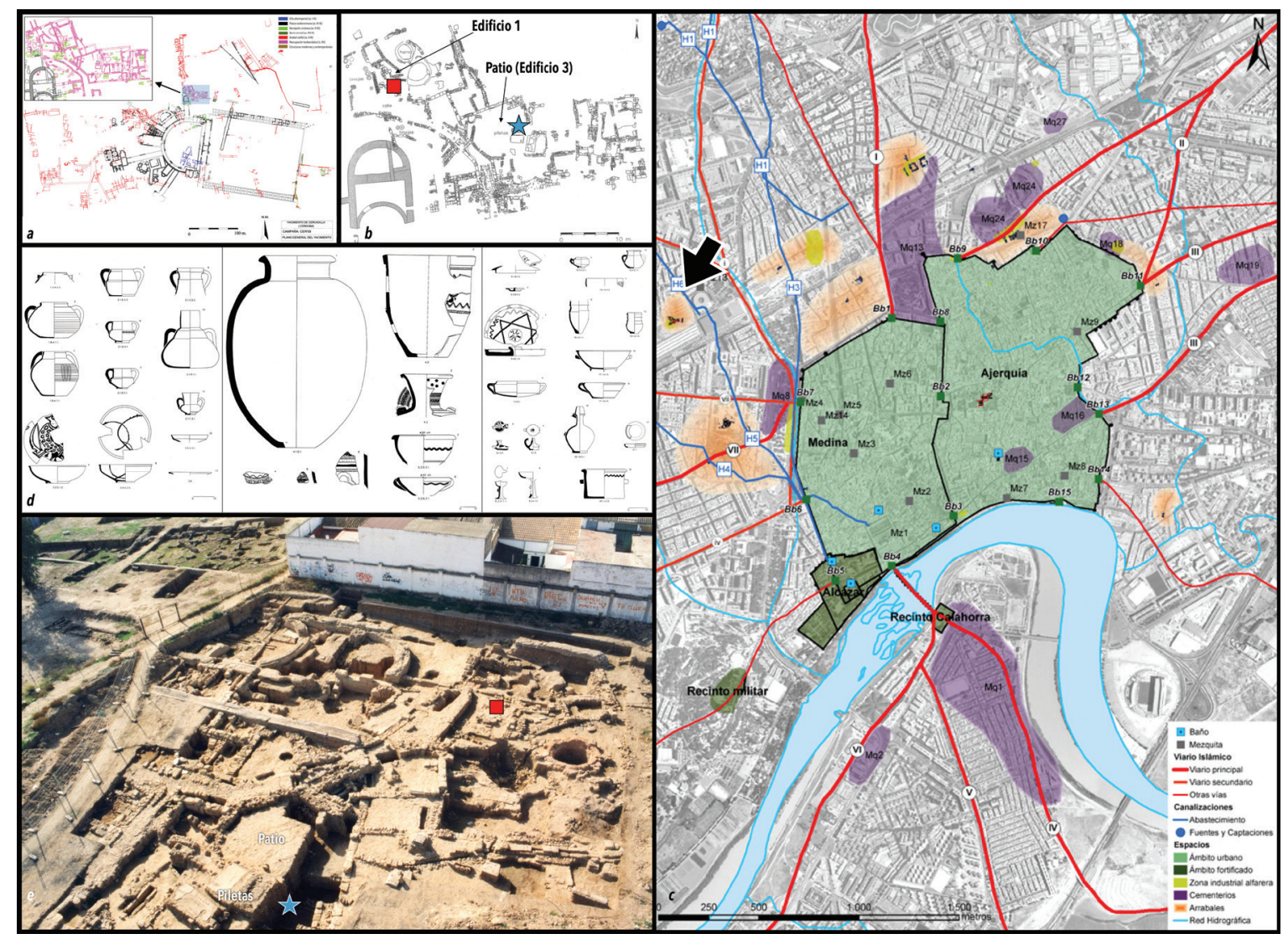

Fig. 2. Fase de reocupación tardoandalusí (s. XII) de Cercadilla: a) Localización sobre el plano general (rectángulo sombreado); b) Planta con señalización del contexto de recuperación del basurero U.E. 73 (estrella azul) y de la valva de vieira (cuadrado rojo); c) Situación de la unidad de poblamiento (flecha negra) en el espacio periurbano de la Qurțuba tardoandalusí (modificado a partir de LEÓN y BLANCO, 2010: Fig. 369). Obsérvese el carácter aislado de la ocupación de Cercadilla en este momento; d) Grupos formales cerámicos recuperados (modificado a partir de FUERTES, 2009); e) Panorámica del complejo doméstico y agroindustrial ( $M^{a}$. del C. Fuertes).

construcción, vida y abandono de este complejo residencial y productivo debe circunscribirse al s. XII (FUERTES, 2006).

\section{ANÁLISIS ARQUEOZOOLÓGICO}

A lo largo del presente epígrafe se presenta el estudio de los restos arqueofaunísticos derivados de la última fase de ocupación medieval de Cercadilla, cuyos rasgos principales acaban de ser introducidos. En primer lugar, se resumen los protocolos metodológicos de análisis arqueozoológico empleados; en segundo lugar, atendemos a los rasgos definitorios de los contextos arqueológicos de procedencia del material para, finalmente, exponer los resultados recabados.

\subsection{Métodos}

La metodología empleada aparece extensamente desarrollada en GARCÍA GARCÍA (2019). De manera sintética, se puede señalar que la identificación taxonómica fue realizada empleando la colección de referencia propia del primer autor. Para la cuantificación de la información arqueozoológica recurrimos principalmente al Número de Restos Identificados (NRI), que fue empleado como la medida básica para el cálculo de los demás parámetros de estudio. El examen de la frecuencia con que aparecen representadas las distintas regiones anatómicas se realizó siguiendo en sus líneas fundamentales, aunque con numerosas modificaciones, el procedimiento analítico desarrollado por $\mathrm{T}$. O'CONNOR (2000, 2003). Por su parte, la edad 
aproximada de sacrificio de los animales fue calculada mediante la observación tanto del estado de fusión de las epífisis, como del de erupción de las piezas dentales y desgaste de las superficies de oclusión de las mismas. Finalmente, para el análisis biométrico empleamos la técnica de log ratio (ALBARELLA, 2002; MEADOW, 1999; SIMPSON et al., 1960) con el objeto de aumentar el nivel de resolución de la identificación taxonómica de los restos examinados.

\subsection{Procedencia del material objeto de estudio}

El material arqueofaunístico se deriva de dos contextos arqueológicos. El primero de ellos se trata de un único depósito documentado en un gran espacio abierto denominado XII.13, de unos $80 \mathrm{~m}^{2}$, asociado a uno de los edificios (Edificio 3) que conforman el complejo doméstico y agroindustrial de época tardoandalusí (Figs. 2b y 2e). La secuencia estratigráfica del ambiente XII.13 permitió documentar dos fases constructivas principales, si bien ambas se enmarcan en un horizonte cronológico que siempre remite al s. XII a juzgar por el material cerámico y numismático recuperado (FUERTES, 2006). Durante la primera fase, parece que este ambiente -identificado como un patio debido a la ausencia de tejas recuperadas en su nivel de frecuentaciónrecibiría agua a través de una canalización, que la conduciría hasta un espacio semisubterráneo abierto de más de $1 \mathrm{~m}$ de profundidad. Sin embargo, en un segundo momento, este espacio fue colmatado con tierra, piedras y basura inorgánica (cerámica) y orgánica, posiblemente con la intención de nivelar el terreno con anterioridad a la construcción de una serie de piletas de decantación. De este relleno, denominado U.E. 73 , se derivan los restos animales que conforman la principal muestra arqueofaunística analizada.

El segundo de los depósitos arqueológicos fue identificado como un nivel de ocupación (es decir, un suelo) excavado en una de las estancias de carácter doméstico que conforman el Edificio 1, situado en la zona norte del mismo complejo estructural tardoandalusí (Figs. 2b y 2e). En este caso, y a diferencia de la muestra del relleno U.E. 73, no nos encontramos ante un conjunto de restos óseos interpretables como residuos de alimentación, sino que se trata de un espécimen animal aislado cuyo interés reside tanto en su contexto de procedencia como en las características particulares que presenta y que lo definen como un artefacto con unas connotaciones socioculturales y simbólicas específicas.

Así pues, conviene enfatizar que el material arqueofaunístico estudiado fue recuperado de depósitos arqueológicos bien contextualizados en términos estratigráficos y, por lo tanto, no afectados por eventos de redeposición o intrusión de material (ALBARELLA, 2015). Las características de dichos depósitos y la presencia en ellos de material tanto cerámico como numismático previamente investigado que, en el marco de la Qurtuba tardoandalusí, ofrece cronologías relativas precisas para la formación de dichos contextos, permite atribuir un elevado grado de calidad informativa a los datos arqueozoológicos examinados, garantizando la correcta contextualización en términos temporales de los patrones que reflejan.

\subsection{Resultados}

La principal muestra arqueofaunística esto es, la derivada del basurero U.E. 73- está conformada por un total de 183 restos identificados (NRI) (Tabla 1, Fig. 3). En términos taxonómicos, el conjunto aparece claramente dominado por restos de suidos Sus sp. (cerdo/ jabalí), presentando una frecuencia muy por encima de la que exhibe el ganado ovino, cabrío y vacuno, que normalmente predomina en los conjuntos arqueofaunísticos peninsulares de época medieval. Este resultado refleja por sí mismo una situación particular en relación a otros casos de estudio examinados hasta la fecha derivados no solo de contextos andalusíes (GARCÍA GARCÍA, 2019), sino también de contextos cristianos en donde los suidos rara vez aparecen tan abundantemente representados (GRAU, 2015). Por esta razón, nuestra atención queda circunscrita de 
Tabla 1. Frecuencia absoluta y relativa de las especies animales identificadas (Número de Restos Identificados). La categoría "OVA+CAH+O/C» incluye los restos identificados a nivel específico (oveja y cabra) y a nivel de subfamilia Caprinae, por lo que sus valores no se incluyen en la cuantificación total.

\begin{tabular}{|l|c|c|}
\hline \multicolumn{1}{|c|}{ ESPECIE } & NRI & $\%$ \\
\hline Oveja (Ovis aries) & 11 & 6 \\
\hline Cabra (Capra hircus) & 6 & 3 \\
\hline Caprinos (Ovis/Capra) & 15 & 8 \\
\hline $\begin{array}{l}\text { Total caprinos } \\
\text { (OvA+CAH+O/C) }\end{array}$ & $(32)$ & $(18)$ \\
\hline Vacuno (Bos taurus) & 23 & 13 \\
\hline Suidos (Sus sp.) & 103 & 58 \\
\hline Équidos (Equus sp.) & 12 & 7 \\
\hline Perro (Canis familiaris) & 10 & 6 \\
\hline Ciervo (Cervus elaphus) & 3 & 2 \\
\hline Total & $\mathbf{1 8 3}$ & $\mathbf{1 0 0}$ \\
\hline
\end{tabular}

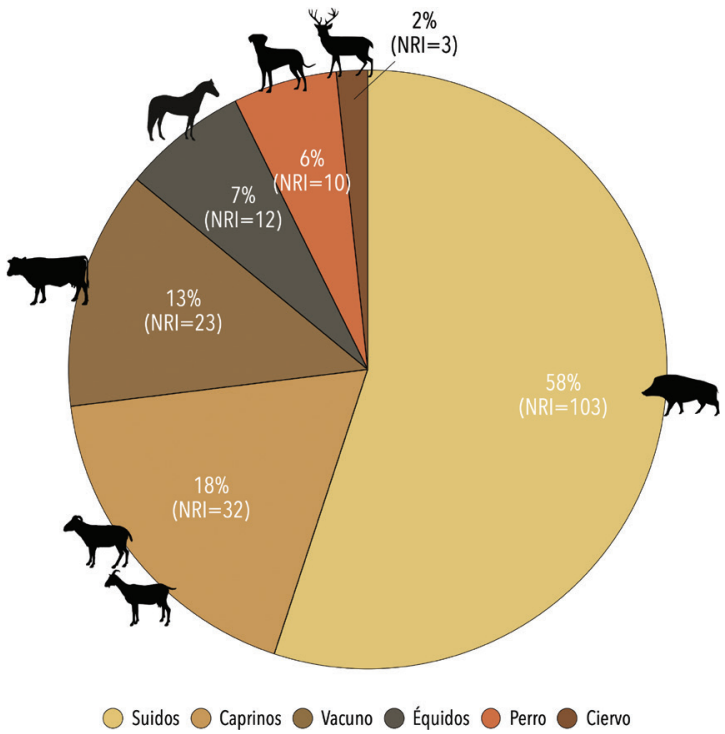

Fig. 3. Frecuencia absoluta y relativa de las especies animales identificadas (Número de Restos Identificados). NRI total=183. El grupo de "Caprinos» incluye los restos identificados a nivel específico y a nivel de subfamilia Caprinae. Datos derivados de la Tabla 1.

manera prioritaria a la exposición de los resultados obtenidos a partir del análisis del grupo taxonómico de los suidos.
En relación al patrón de distribución anatómico (Tabla 2, Fig. 4), se debe ante todo destacar la presencia predominante de los huesos que conforman el cuarto superior del miembro anterior (la región conocida como paleta), principalmente de la zona distal de la escápula y, sobre todo, del húmero, elemento este último que, con diferencia, domina la muestra. A cierta distancia aparecen representadas la zona distal del fémur y la región del acetábulo de la pelvis, ambos elementos derivados del cuarto superior del miembro trasero (jamón), estando tanto la región craneal como los extremos apendiculares (metápodos y falanges) claramente infrarrepresentados. Así pues, si asumimos que los fragmentos de húmeros y fémures distales examinados se derivan de porciones anatómicas completas, se concluye que dichos restos representan un mínimo de doce paletas y cinco jamones, respectivamente.

Aunque algunos de los elementos óseos mencionados se encuentran entre los más robustos del esqueleto debido a su elevada densidad estructural, sorprende la escasa presencia de otros igualmente densos que suelen presentar buenas tasas de preservación en contextos arqueológicos, tales como la tibia distal, el radio proximal, la mandíbula o las piezas dentales. Por este motivo, asumimos que la afección de los factores de alteración tafonómica que conllevan la destrucción posdeposicional de los restos no es el factor determinante para explicar la composición anatómica de los suidos en la muestra que nos ocupa, sino que parece más bien responder a una dinámica cultural bien definida consistente en la introducción de aquellas porciones anatómicas de mayor contenido cárnico (jamones y, particularmente, paletas) al espacio de consumo del que fueron recuperados.

El escaso número de piezas dentales en secuencia mandibular dificulta el análisis de la edad de sacrificio, al ser los restos más informativos para el examen de este parámetro de estudio. Aunque solo fue posible atribuir tres fragmentos mandibulares a un grupo de edad específico, dos de ellos presentan una información que resulta de particular interés. 


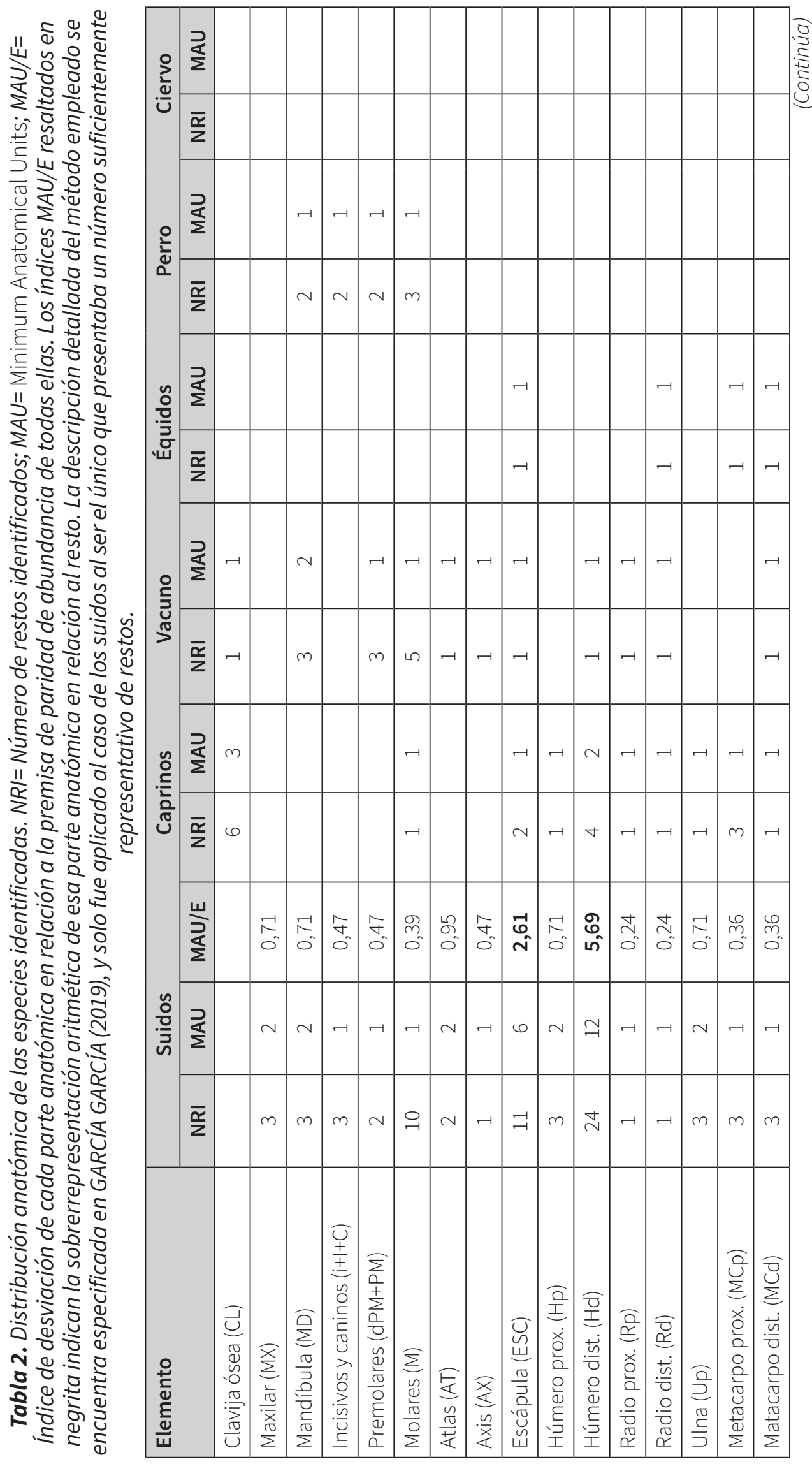




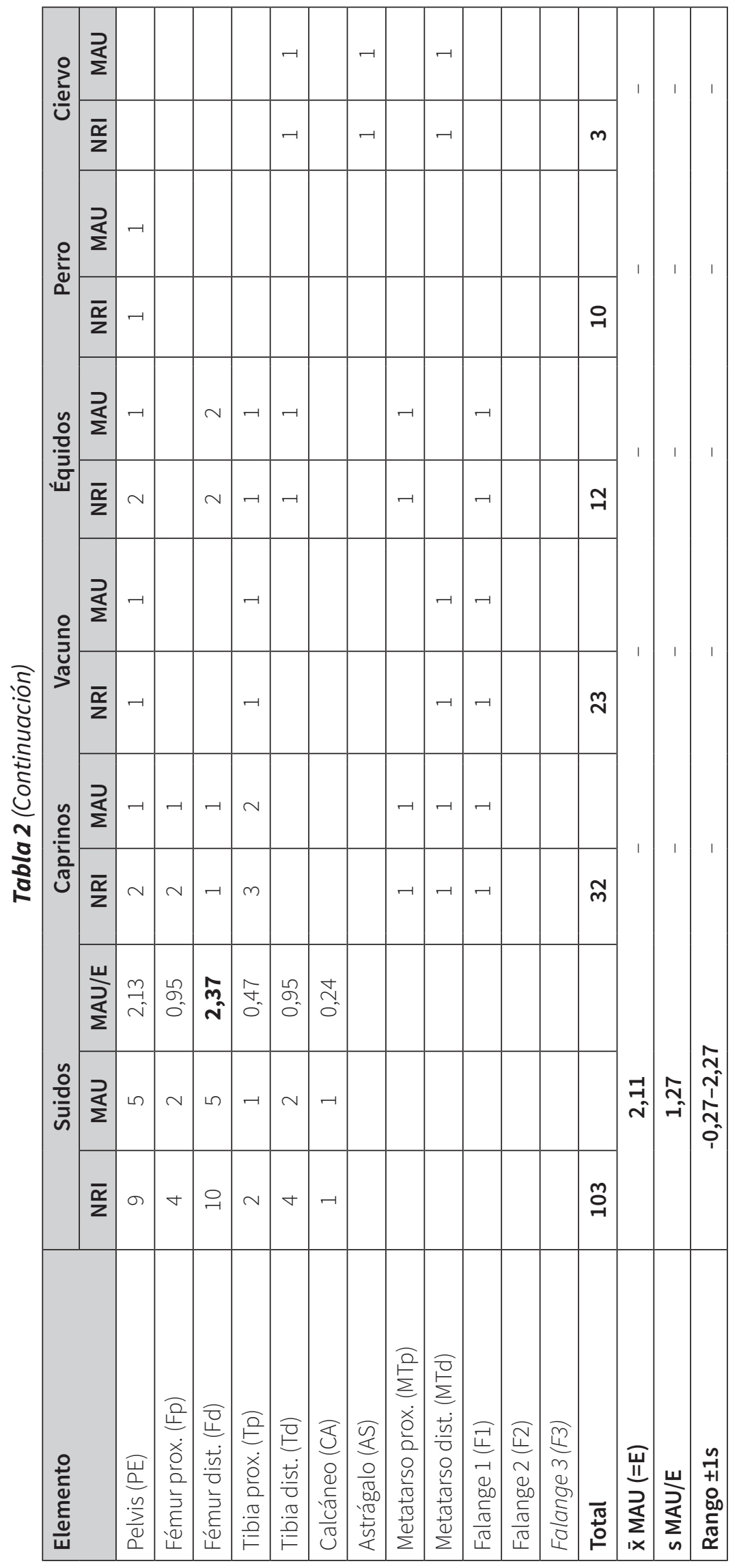




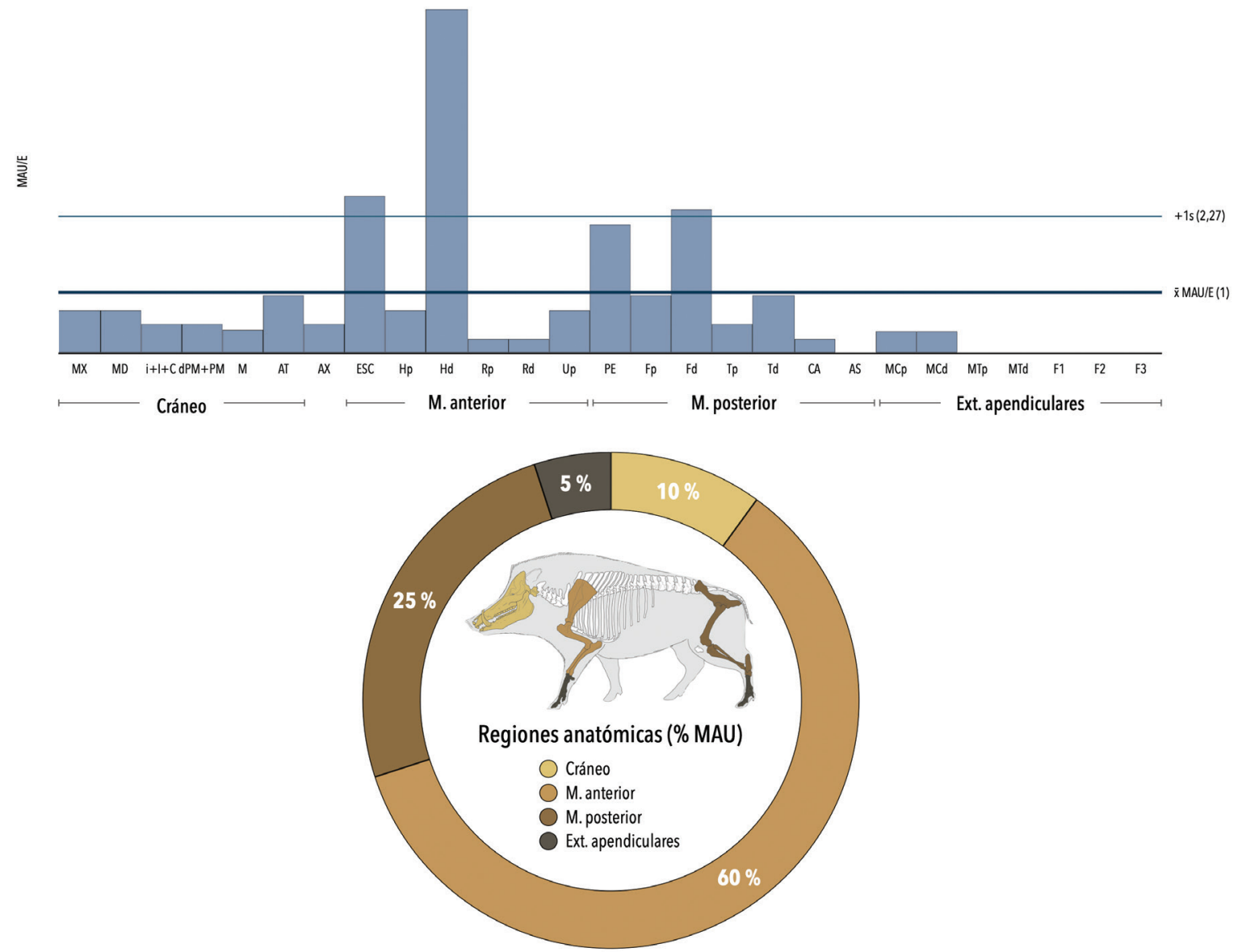

Fig. 4. Distribución anatómica de los suidos. Arriba: abundancia de cada elemento anatómico en función de su índice MAU/E; abajo: abundancia relativa (\%MAU) de las principales regiones anatómicas. La categoría «Ext. apendiculares» incluye las falanges y los metápodos. La descripción detallada del método empleado se encuentra especificada en GARCÍA GARCÍA (2019). Las abreviaciones del desglose anatómico aparecen desarrolladas en la Tabla 2.

El primero corresponde a una hemimandíbula completa de un espécimen infantil de escasos meses de vida que presentaba un estado inicial de desgaste del $\mathrm{dP}_{4}$, el $\mathrm{M}_{1}$ en cripta y un aspecto rugoso de la cortical del hueso (Fig. 5a). El segundo pertenece a un animal adulto (abatido durante su tercer año de vida) que, al margen de esta consideración, presentaba una patología denominada «hipoplasia lineal del esmalte» en el segundo de sus molares (Fig. 5b), reconocible por la presencia de surcos o líneas sobre la superficie dental cuyo origen debe relacionarse con una deficiencia en el espesor del esmalte como consecuencia de episodios de estrés fisiológico provocados por enfermedades, deficiencias nutricionales o exposición a temperaturas extremas durante la formación de la corona (COLYER, 1936; DOBNEY et al., 2004, SARNAT y MOSS, 1985). Según DOBNEY y ERVYNCK (1998), el principal factor causal del tipo de hipoplasia observado (una única línea en el $M_{2}$ ) se vincula a un periodo de desnutrición entre el primer y séptimo mes de vida del animal, comúnmente durante el destete. Es decir, la patología documentada generalmente resulta de la interferencia humana durante la fase de crecimiento del animal, por lo que su presencia suele ser más habitual en individuos criados en cautividad (cerdos domésticos) que en salvajes (jabalíes), una observación válida tanto para poblaciones arqueológicas como actuales (DOBNEY et al., 2004, 2007). Este rasgo representa un indicio que deberá ser tomado en consideración a la hora de interpretar la 

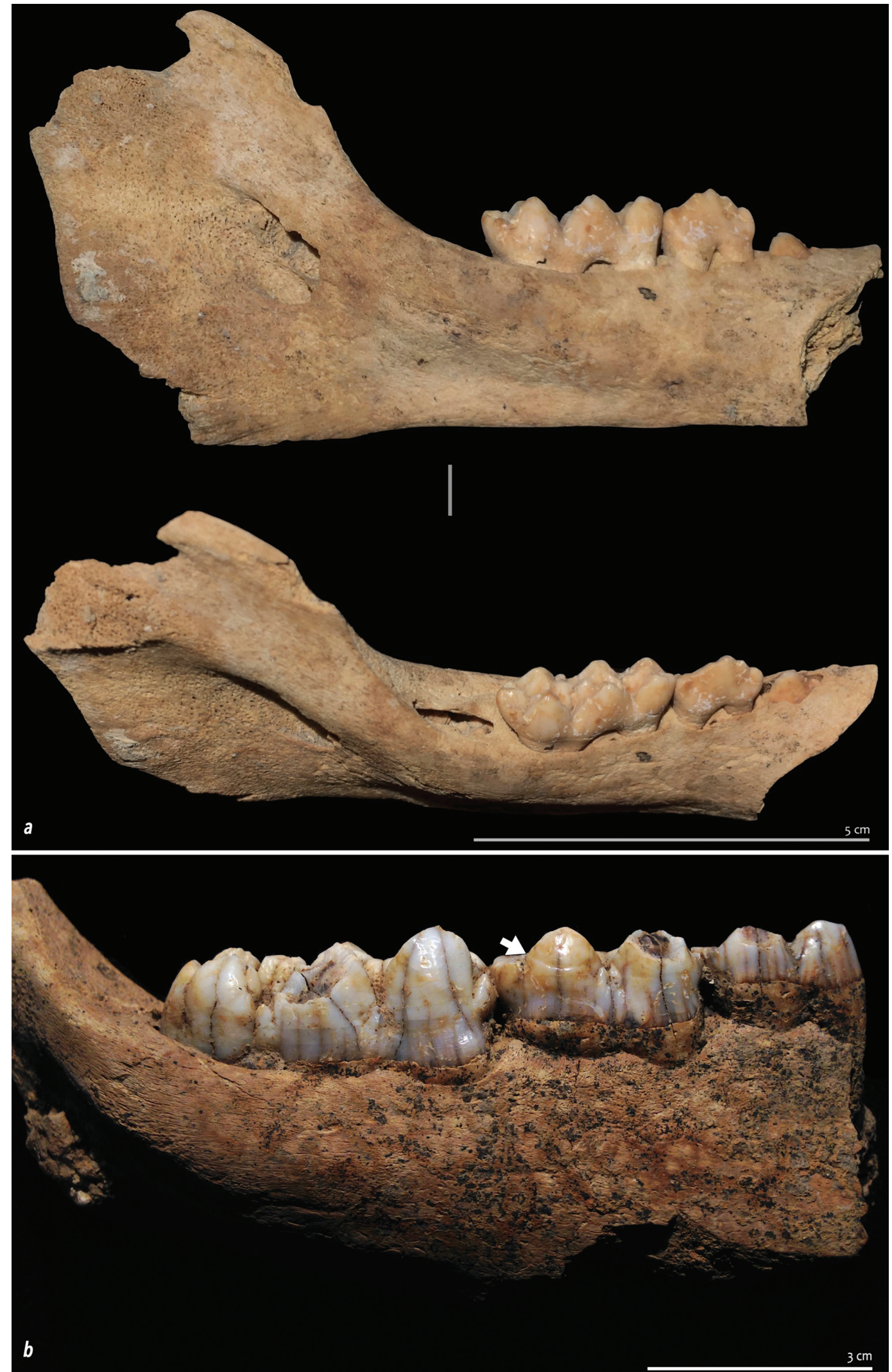

Fig. 5. Mandíbulas de suidos: a) la dentición decidua indica que se trata de un individuo infantil; b) a destacar la hipoplasia linear del esmalte en el $M_{2}$ (indicada con una flecha). 
Tabla 3. Edad estimada de abatimiento de los suidos según el estado de fusión de los elementos poscraneales, reflejado en los porcentajes de huesos pertenecientes a individuos adultos ( $A=$ huesos soldados) y juveniles ( $J=$ huesos no soldados) en cada uno de los grupos de edad propuestos por SILVER (1969).

\begin{tabular}{|c|c|c|c|}
\hline $\begin{array}{l}\text { GRUPO DE FUSIÓN Y } \\
\text { RANGO DE EDAD }\end{array}$ & ELEMENTO & A & $\mathrm{J}$ \\
\hline \multirow[t]{5}{*}{ Temprana (<1,5 años) } & ESd & 8 & \\
\hline & $\mathrm{Hd}$ & 19 & 1 \\
\hline & $\mathrm{Rp}$ & & \\
\hline & F2p & & \\
\hline & $\%$ media juvenil & 4 & \\
\hline \multirow[t]{5}{*}{ Media (1,5-2,5 años) } & $\mathrm{MPd}$ & 1 & 1 \\
\hline & F1p & & \\
\hline & $\mathrm{Td}$ & 3 & 1 \\
\hline & CA & & \\
\hline & $\%$ media juvenil & 33 & \\
\hline \multirow[t]{7}{*}{ Tardía (2,5-3,5 años) } & Up & & 3 \\
\hline & $\mathrm{Hp}$ & 3 & \\
\hline & $\mathrm{Rd}$ & 2 & \\
\hline & $\mathrm{Fp}$ & 1 & 1 \\
\hline & $\mathrm{Fd}$ & 6 & 1 \\
\hline & $\mathrm{Tp}$ & & 1 \\
\hline & $\%$ media juvenil & 33 & \\
\hline \multicolumn{2}{|c|}{$\%$ media animales jóvenes } & 17 & \\
\hline $\mathrm{N}$ & & 52 & \\
\hline
\end{tabular}

evidencia arqueofaunística disponible. Por último, el tercer resto mandibular se atribuyó a un individuo subadulto sacrificado a lo largo de su segundo año de vida.

Al margen de los datos dentales, el examen del estado de fusión epifisaria de los huesos poscraneales ofrece igualmente información sobre la edad de sacrificio (Tabla 3). Aunque la resolución de este parámetro es menos precisa que la obtenida a partir de las secuencias mandibulares (O'CONNOR, 2006), la escasa frecuencia de huesos no soldados (17\%) indica una presencia mayoritaria -si bien, como acabamos de ver, no exclusiva- de animales adultos de edad avanzada. Este resultado contrasta con el obtenido para el conjunto de restos de suidos de la fase emiral del mismo espacio de Cercadilla, donde el $56 \%$ de los elementos poscraneales presentaban sus epífisis no soldadas (GARCÍA GARCÍA, 2019), reflejando una presencia mayor de animales inmaduros y subadultos congruente con una práctica de explotación tradicional de porcino doméstico a escala local.

El último de los parámetros de estudio arqueozoológico explorados concierne al análisis biométrico. Para ello, procedimos al examen comparativo mediante la técnica de log ratio del tamaño de los huesos poscraneales ${ }^{7}$ y de la anchura de los molares inferiores de la muestra

\footnotetext{
7. A pesar de que, como demostró S. DAVIS (1996), las medidas tomadas a lo largo de cada uno de los planos anatómicos presentan mejor correlación cuando son comparadas entre sí, la escasa entidad numérica de las muestras disponibles (particularmente de la aquí examinada del s. XII) nos obligó a considerar conjuntamente las medidas derivadas de los tres ejes esqueléticos.
} 

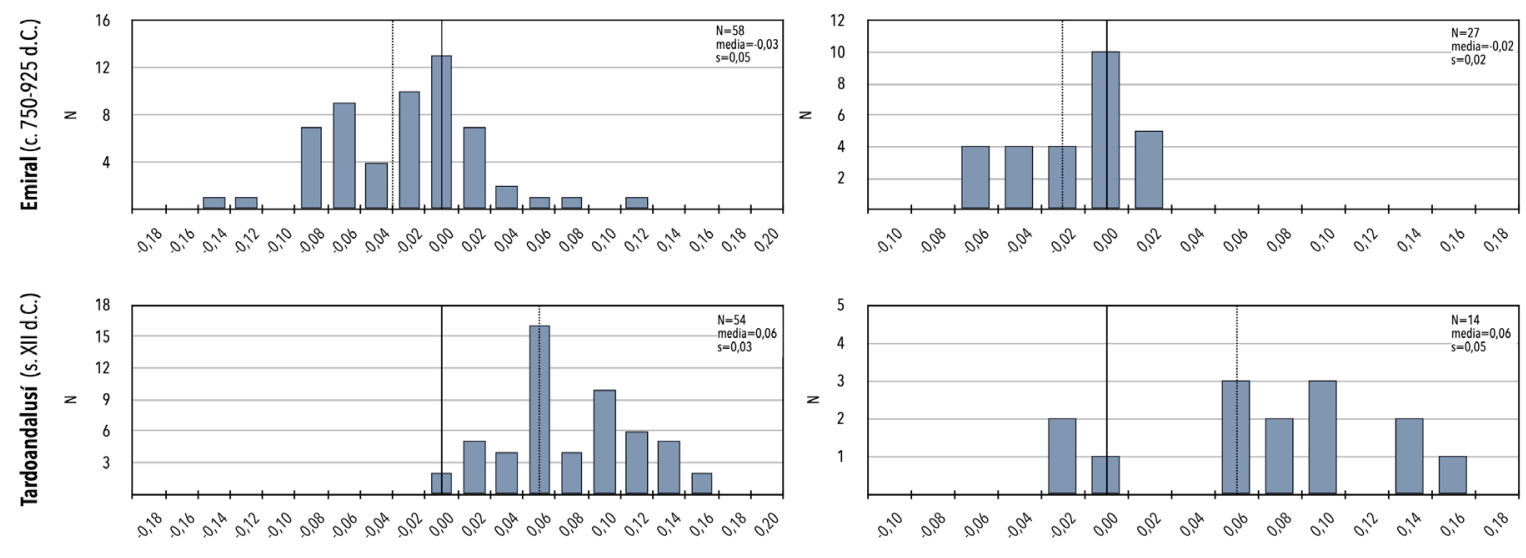

Huesos poscraneales

Anchura molares inferiores

Fig. 6. Diagramas de log ratio de las medidas de los huesos poscraneales (combinando todas las medidas con independencia del eje esquelético) y de las anchuras de los molares inferiores de suidos en las muestras de época emiral y tardoandalusí de Cercadilla. La línea vertical continua representa el estándar, mientras que la discontinua indica la medida de cada conjunto de datos.

de suidos recuperados de los niveles emirales de ocupación (interpretados como porcino doméstico ${ }^{8}$ ), de un lado, y la de época tardoandalusí, de otro (Fig. 6). La diferencia métrica observable entre ambas muestras es estadísticamente muy significativa (prueba U de Mann-Whitney $p<0,001$ ) tanto para los elementos poscraneales como para los dentales, revelando el mayor tamaño y robustez de los suidos del s. XII en relación a los de época emiral. Aunque no es posible descartar de plano que este patrón biométrico sea la consecuencia de diferencias diacrónicas relativas a la composición sexual o a la edad de sacrificio de los suidos, la magnitud con que se manifiesta permite apuntar la posibilidad de que al menos una parte de los suidos derivados del conjunto del s. XII perteneciesen a jabalí. Con todo, como trataremos de demostrar, la identificación taxonómica específica de estos restos representa, en el marco de la presente contribución, un problema secundario que, a pesar de ello, será abordado a la luz de los resultados de los análisis biomoleculares que esperamos desarrollar próximamente.

Dejando a un lado la asociación de restos arqueofaunísticos dominada por suidos de la que nos venimos ocupando, ya hemos señalado el interés que presenta el hallazgo de un resto faunístico aislado, de características muy

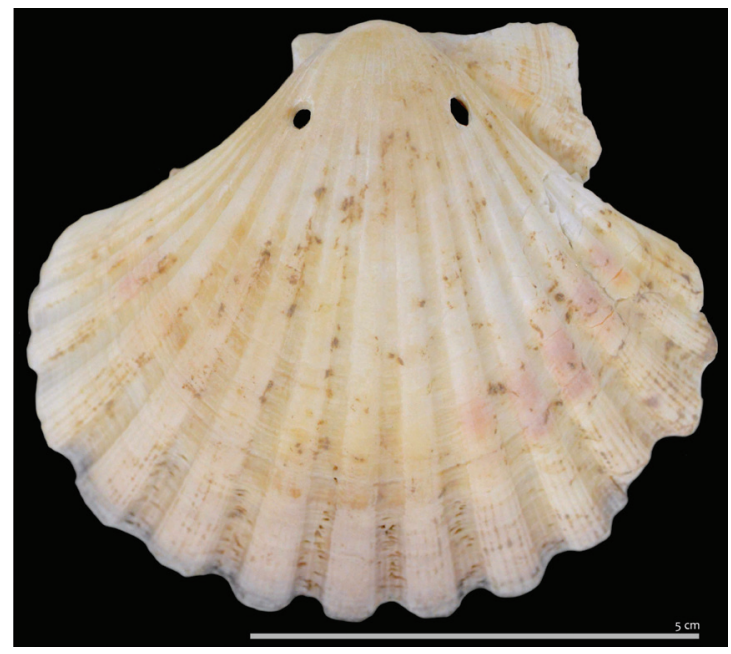

Fig. 7. Valva derecha de vieira Pecten maximus con dos perforaciones de origen antrópico en la zona del umbo.

específicas, sobre un suelo o nivel de ocupación del mismo complejo estructural tardoandalusí. Concretamente, se trata de una valva derecha (la cóncavo-convexa) de vieira Pecten sp. en la que se identificaron dos perforaciones de origen claramente antropogénico en la zona del borde del umbo (Fig. 7). Aunque la distinción entre las dos principales especies de Pecten (P. maximus y $P$. jacobaeus) no siempre resulta sencilla debido a las semejanzas que presentan tanto en

8. Los perfiles de sacrificio indican la presencia de individuos de diferentes edades -incluyendo un grupo de animales osteológicamente inmaduros abatidos durante su primer año de vida-, mientras que la distribución esquelética se define por la frecuencia en términos similares de todas las partes anatómicas, si bien destaca la dominancia que ejerce la región craneal. 
dimensiones como en estructura, sobre la base de la forma de las costillas radiales y de la zona del borde (WAGNER, 1991) atribuimos este espécimen a P. maximus ${ }^{9}$. Tanto su localización como el hecho de haber sido alterado a fin de convertirlo en un artefacto le otorgan un indudable interés por las connotaciones socioculturales que presenta.

En definitiva, el examen de los restos animales derivados de la fase tardoandalusí de ocupación de Cercadilla ha reportado un conjunto de resultados que, desde múltiples ángulos de análisis, permiten arrojar luz sobre diversos aspectos relacionados tanto con las estrategias de aprovechamiento y consumo de recursos de origen animal por parte del grupo humano residente en este espacio, como con la posible identidad en términos socioculturales y étnicos de esta comunidad, labor a la que dedicaremos la siguiente sección.

\section{DISCUSIÓN}

La discusión en términos interpretativos de los datos recabados se estructurará en dos apartados, habida cuenta de la diferente naturaleza de la información proporcionada por el estudio del basurero U.E. 73 y la vieira perforada recuperada del suelo de ocupación del Edificio 1. Atenderemos primeramente al examen de las formas de gestión de los suidos sobre la base de los datos recabados a partir del análisis de la composición anatómica y la edad de sacrificio, así como a las implicaciones socioculturales que presenta el hábito de alimentación basado en el consumo de este taxón. En segundo lugar, desplazaremos el foco de atención a la discusión del resto malacológico cuya procedencia y características requieren de un tratamiento independiente.

\subsection{El conjunto de restos de suidos del basurero U.E. 73}

El principal elemento a destacar en relación al basurero U.E. 73 es la abundante presencia -excepcionalmente elevada en el panorama arqueozoológico ibérico de época medieval-, de restos de suidos que podemos interpretar sin demasiado riesgo como el resultado del consumo de la carne que contenían por parte del grupo residente en este espacio periférico de la Qurțuba andalusí durante el siglo XII. Así pues, los datos manejados presentan un enorme interés al reflejar la práctica de un hábito de consumo alimentario centrado sobre un producto animal que, tanto en su variedad doméstica como salvaje, es considerado ilícito (harām) en la normativa dietética que conforma la ortopraxis del islam medieval (BENKHEIRA, 1995, 2002, 2006; COOK, 1986; LOBBAN, 1994; VIRÉ, 1986).

Sin embargo, antes de atender a las cuestiones de orden más puramente sociocultural relacionadas con esta circunstancia, se impone considerar los patrones de distribución anatómica y de mortalidad observados, dado que ambos parámetros contienen información relevante sobre las posibles formas de gestión y aprovechamiento de la cabaña porcina.

\subsubsection{Formas de explotación y gestión de las carcasas: composición anatómica y edad de sacrificio}

La composición anatómica de la muestra revela una predominancia muy evidente del esqueleto apendicular, constatándose la presencia mayoritaria de dos porciones cárnicas bien definidas como son la paletay, en menor medida, el jamón. Este resultado sugiere la existencia de un patrón de distribución de los productos animales caracterizado por la introducción al espacio de consumo de dos de las partes anatómicas más productivas en términos cárnicos que, presumiblemente, habrían sido previamente procesadas en un lugar externo a esta zona de hábitat. Así parece indicar igualmente la escasa frecuencia de las regiones que habitualmente se asocian con los desechos de las primeras labores del proceso de carnicería (concretamente el cráneo y los extremos apendiculares), lo que podría efectivamente reflejar que el sacrificio y primer despiece no se practicaron in loco y que en el depósito

9. Identificación igualmente compartida por el experto malacólogo E. González (Universidad de León). 
examinado mayoritariamente se acumularon residuos de consumo culinario.

En relación a la edad de sacrificio, hemos visto cómo la mayor parte de los restos pertenecen a individuos adultos, siendo minoritaria la presencia de especímenes infantiles, juveniles o subadultos. Es importante señalar que este resultado difiere significativamente del que generalmente se atribuye a la gestión local y al aprovechamiento a nivel doméstico de porcino, que tiende a manifestarse en el registro arqueofaunístico en una mayor contribución de individuos abatidos a edad más temprana (REDDING, 1991) tal y como se constata, por ejemplo, durante la fase emiral de ocupación.

Así pues, los resultados de ambas líneas de análisis apuntan a una situación particular que difícilmente puede interpretarse como el resultado de un tipo de explotación «habitual» de porcino doméstico, criado in loco y preparado para su consumo en el mismo espacio de ocupación. En este caso, los datos parecen indicar que los restos examinados proceden de la importación de piezas cárnicas derivadas de animales de edad avanzada y que previamente habrían sido procesadas en un lugar externo al espacio de consumo del que se recuperaron. En coherencia con la interpretación taxonómica propuesta a partir de la información biométrica pudieron pertenecer, al menos en parte, a jabalí. Dada la presencia histórica, persistente a día de hoy, de piaras en el entorno próximo al emplazamiento de Cercadilla, una posible lectura interpretativa pasaría por asumir que la evidencia aquí expuesta refleje el consumo de carne de jabalíes adultos cazados - posiblemente mediante trampeo- en las inmediaciones del asentamiento, cuyas carcasas fueron procesadas off-site, introduciéndose únicamente las partes anatómicas más productivas (paletas y jamones) al espacio de hábitat en que fueron recuperados.

Sin embargo, algunos de los datos extraídos del examen de los escasos restos dentales presentes en la muestra no encuentran fácil acomodo e, incluso, resultan en cierta medida incongruentes con esta hipótesis de trabajo. Concretamente nos referimos, de un lado, a la hemimandíbula perteneciente a un individuo infantil de pocos meses de edad y, de otro lado, al fragmento mandibular que presentaba hipoplasia linear en el segundo molar. Aun considerando que la representatividad es limitada al derivarse únicamente de dos individuos, su constatación permite ofrecer una segunda propuesta interpretativa a la luz de una serie de referencias etnográficas que, a nuestro juicio, presentan un notable valor heurístico para dar sentido a este conjunto de evidencias.

C. COON (1931), en su descripción de las bases económicas de varias poblaciones rifeñas del norte de Marruecos que visitó durante la década de los años 20, destaca cómo algunos de estos grupos rurales criaban suidos que, sin embargo:

«[...] are not the descendants of the any long-domesticated breed, but are bred from individuals caught in traps on the mountains; seldom does a litter have a domestic history of more than two or three generations. When all have been killed off, more are caught and bred. Thus swine domestication is, or was, either in an incipient stage or in the last stage of decadence. The animals are kept in the house, but are confined in a separate room, the door of which is closed at night. In the daytime they are taken out on the mountain slopes to feed on acorns and roots. When they are slaughtered, no hallala is performed» (COON, 1931: 41).

Por otro lado, S. BÖKÖNYI (1988: 207) apunta que, con anterioridad a la I Guerra Mundial, cuando extensos grupos de cristianos armenios habitaban en el Imperio otomano, era una práctica relativamente común «[...] to capture the piglets of wild swine, to rear and later to slaughter them because no pig keeping could develop in a Moslem state».

El interés fundamental de estos referenciales etnográficos radica en que ambos se derivan de contextos sociales islamizados como son el Magreb y el Imperio otomano. Si, en el caso armenio, el consumo de suidos no supone el incumplimiento de la normativa dietética del grupo al tratarse de una comunidad cristiana, el aprovechamiento de jabalí por parte de grupos 
rifeños sí refleja una «islamización de baja intensidad» en lo referente a los patrones alimentarios de ciertas poblaciones rurales magrebíes (BENKHEIRA, 1997). Aunque lo realmente relevante desde nuestro punto de vista es que el factor determinante que explica la adopción de esta forma de explotación de porcino es la existencia de un ambiente sociocultural y político que no favorece el desarrollo de una práctica «normal» (doméstica) de aprovechamiento de estos animales (SIMOONS, 1994: 33). A tenor de toda la información histórica disponible, no parece arriesgado sugerir que este sería, precisamente, el panorama social que caracterizaría a la Qurtuba del s. XII tras cuatro siglos de profunda islamización a todos los niveles.

En consecuencia, sugerimos como hipótesis de trabajo la posibilidad de que al menos parte de los restos objeto de estudio se deriven de porcino gestionado localmente, quizá en régimen de semilibertad, por el grupo residente en este sector periurbano y relativamente aislado de la Qurtuba tardoandalusí. Esta propuesta permite explicar la concurrencia en un mismo depósito de restos biométricamente atribuibles a jabalí, de un lado, y de un individuo de pocos meses y otro con hipoplasia dental que podrían interpretarse como ejemplares criados localmente, de otro. Igualmente, encontraría fácil acomodo con los resultados derivados del examen de la frecuencia anatómica que, como hemos visto, sugieren que la primera fase del proceso de carnicería consistente en el despiece de las carcasas -y quizá también el sacrificio - se realizase en un lugar externo a la zona de hábitat. Por ello, resulta plausible plantear que el grupo residente en Cercadilla optase por (o se viese forzado a) abatir a estos animales en un lugar externo y alejado de espacios de ocupación, en donde previsiblemente fuesen descartadas las partes anatómicas menos productivas que aparecen infrarrepresentadas en la muestra.

Al hilo de esta idea, observaciones actuales realizadas por uno de nosotros (M. MORENO GARCÍA) sobre el consumo -siempre puntual y fuera de la norma general de alimentaciónde jabalí en la región Jebala del Rif occidental constituyen un paralelo revelador. Las informaciones recabadas abundan en las impresiones adelantadas por C. COON (1931) que reflejan la consideración, ciertamente particular y a todas luces diferente a la del resto de las especies, de este animal en el Magreb tal y como indican las formas de gestión de sus carcasas, dado que la primera fase de carnicería "can take place either in the forest or in the main square of the village, never in the households as is usually the case with domestic animals» (MORENO, 2004: 330). La correspondencia entre este comportamiento y la composición anatómica del conjunto de restos de suidos de Cercadilla resulta elocuente, por lo que quizá en este caso también debamos apelar a este tipo de consideraciones de orden social a la hora de explicar el tratamiento que recibieron las carcasas de estos animales. Cabría dilucidar, en cualquier caso, el motivo por el que los cuartos superiores de los miembros delantero y trasero aparecen representados en proporciones tan diversas, pudiéndose quizá sugerir la existencia de factores relativos a la distribución horizontal de estos productos cárnicos entre diferentes grupos de consumidores.

En definitiva, el examen de la distribución anatómica y de la edad de sacrificio de este conjunto de suidos revela datos de enorme interés y particularidad que, en nuestra opinión, se corresponden con el hábito de alimentación, igualmente anómalo, que refleja la composición taxonómica de la muestra. A pesar de que los métodos de análisis arqueozoológico aquí empleados no permiten identificar eventuales procesos de preservación de los productos cárnicos, no se debe descartar que los restos de suidos que dominan la muestra no fuesen consumidos en fresco, sino que deriven de la preservación de la carne de paletas y jamones, ya como piezas completas, ya de la carne deshuesada. En cualquier caso, e independientemente del sistema de explotación doméstico, semidoméstico o salvaje de este grupo de animales y de las formas de gestión de sus carcasas, lo que verdaderamente nos interesa destacar es la evidencia que nos informa sobre un comportamiento alimentario enormemente significativo al testimoniar una 

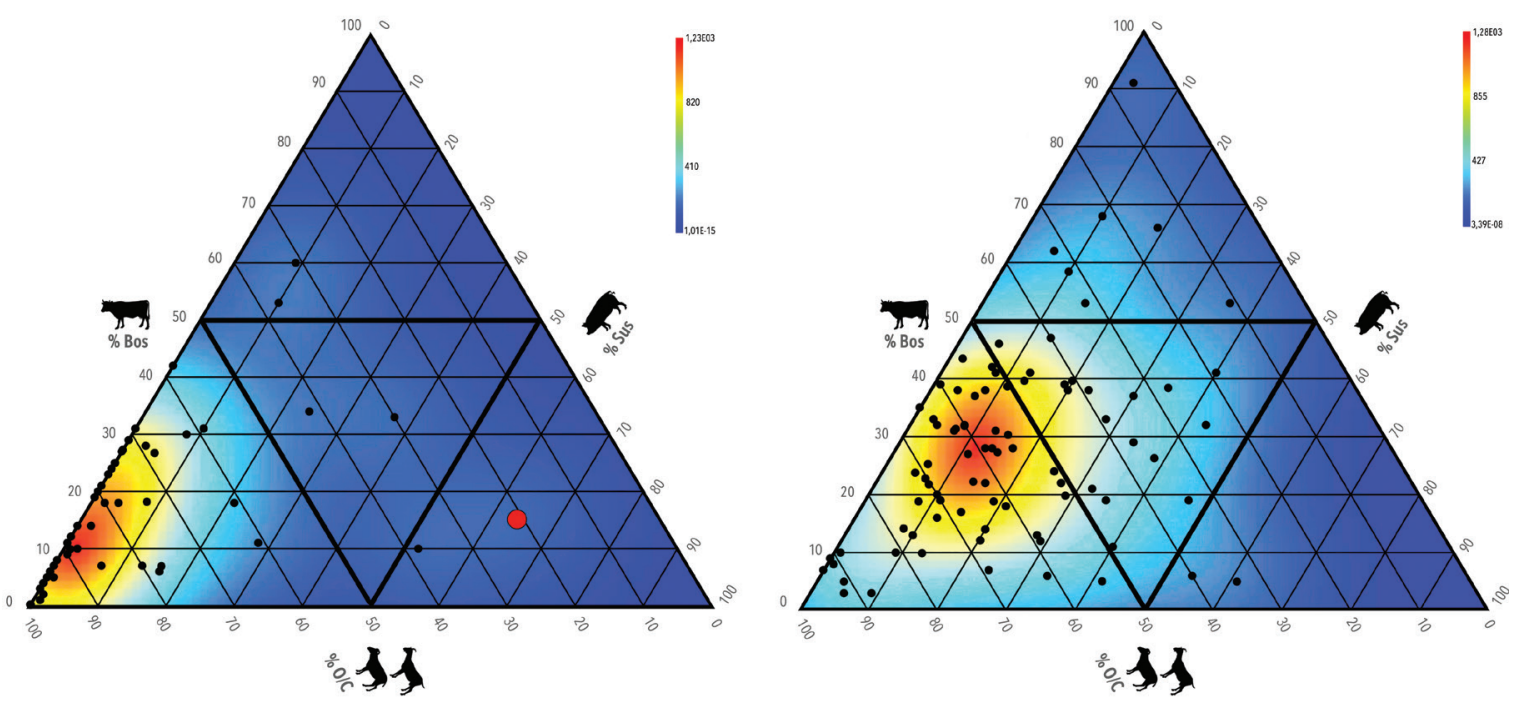

Fig. 8. Gráficos ternarios de frecuencias de vacuno (Bos), caprinos (O/C) y suidos (Sus) en los casos de estudio examinados hasta la fecha de contextos andalusies (izquierda, $N=55$ ) y "cristianos» (derecha, $N=84$, vid. n. 10). Únicamente se consideraron aquellos casos en que el NRI de Bos+O/C+Sus era igual o superior a 100. La muestra tardoandalusí de Cercadilla aparece representada por un círculo rojo en el gráfico de la izquierda.

transgresión evidente de la normativa dietética básica del islam. Por ello, desplazaremos a continuación el foco de atención a la discusión de las posibles implicaciones de orden social y cultural que esta evidencia comporta para evaluar la posible identidad del grupo residente en Cercadilla durante el s. XII.

\subsubsection{Hábitos de alimentación e inferencias socioculturales}

Como venimos señalando, la elevada frecuencia que exhiben los suidos en la muestra recuperada del basurero U.E. 73 resulta del todo anómala en relación al panorama que emerge del registro arqueofaunístico de contextos tanto andalusíes como cristianos medievales. A fin de ilustrar esta observación, en la Fig. 8 se incluyen dos gráficos ternarios que reflejan la frecuencia de los principales grupos taxonómicos (caprinos, vacuno y suidos) en contextos peninsulares tanto andalusíes como «cristianos» ${ }^{10}$ en los que el número total de restos identificados de estos tres taxones era igual o superior a $100^{11}$. Como se aprecia claramente, la situación que ocupa en el gráfico Cercadilla es excéntrica y tendente hacia la esquina inferior derecha, lo que lo convierte en el caso con mayor representación de suidos. Nos encontramos, en consecuencia, ante una asociación de restos animales que nos informa sobre un hábito de alimentación relativamente inédito en el marco histórico investigado y que, como trataremos de demostrar, presenta un notable interés desde un punto de vista social y cultural.

Conviene comenzar enfatizando una vez más que, en el contexto de la normativa dietética del islam medieval, la consideración del consumo de carne de porcino como un acto ilícito (harām) afecta indistintamente tanto a la forma doméstica (cerdo) como a la salvaje (jabalí) de este animal (BENKHEIRA, 1995, 2000, 2002; VIRÉ, 1986), representando además uno de los marcadores culturales más potentes empleados en la demarcación de las fronteras

10. A pesar de su ambigüedad, consideramos operativo el término «cristiano» para referirnos, por simple oposición al marco sociocultural islámico andalusí, a aquellos casos de estudio derivados de contextos de cronología tardoantigua, medieval o moderna «no andalusíes». Con todo, emplearemos entrecomillado el término por hacer aparentemente referencia, en la línea de la conocida crítica de E. MANZANO (1994) a M. ACIÉN (1997, $1^{\text {a }}$ ed., 1994), a un elemento superestructural como es la religión para cualificar una formación social.

11. Los gráficos y los datos en ellos contenidos forman parte de la investigación en que se inserta la presente contribución (GARCíA GARCÍA, 2019). 
culturales que delimitan la comunidad social y religiosa islámica. En opinión de M. BENKHEIRA (1999), autor al que aludiremos repetidamente a lo largo de esta sección por ser el que mayor atención ha dedicado al estudio antropológico de la alimentación en el mundo islamizado, el consumo de carne en dicho contexto cultural presenta una función ritual ligada a la reproducción del vínculo social, motivo por el cual toda ceremonia colectiva que asocie a la totalidad del grupo o solo a ciertos miembros incluye un plato eminentemente cárnico. Lo relevante en relación a esta cuestión, es que el consumo de carne «prohibida» de suidos en un medio social islamizado como puede ser el del Magreb actual, aun siendo excepcional, se encuentra igualmente sujeto a unas reglas sociales específicas dentro de un marco ritual que presenta una intencionalidad esencial, concretamente:

«Manifeste un rejet des croyances et des règles du groupe, una rébellion contre son ordre qui peuvent être interprétés comme une volonté de sortir du groupe [...] il ne paraît pas exagéré de reconnaître au cochon un status d'animal rituel, donc de nourriture rituelle, dans le mesure où consommer sa viande peut être assimilé à un acte blasphématoire» (BENKHEIRA, $1999:$ 92)

En ese mismo trabajo, Benhkeira cualifica el de suidos como un tipo de consumo «transgresivo-agregativo», cuya función es la de construir o reforzar «un petit groupe d'individus qui vit en marge de, et souvent en conflit avec la grande collectivité dont il fait partie» (p. 94). El que se centre exclusivamente sobre el cerdo y el jabalí se debe a que, precisamente, estos animales representan a los ojos de la mayoría de la población el principal demarcador cultural de pertenencia a la comunidad islámica.

Sobre la base de estas consideraciones, y asumiendo que el patrón de alimentación que se refleja en el basurero de Cercadilla representa una transgresión de uno de los pilares esenciales de la ortopraxis del islam medieval, estimamos razonable sugerir la posibilidad de que el grupo responsable de la generación de estos residuos no se incluyese de facto en el orden social islamizado e incluso, siguiendo a Benkheira, que quizá se situase en oposición al mismo. En tal sentido, la situación espacial periférica de este núcleo de población quizá pudo contribuir al mantenimiento de formas de vida «marginales» -en términos sociales y no necesariamente económicos- en relación al modelo islamizado representado por la madina cordobesa.

Así pues, las opciones que se nos presentan a la hora de emplear la información arqueozoológica a disposición para evaluar la posible identidad sociocultural y etnorreligiosa de dicho grupo son múltiples, pero destacamos dos propuestas de interpretación como las más plausibles.

En primer lugar, se puede plantear la posibilidad de que los residentes en este sector relativamente aislado del entorno periurbano cordobés fuesen practicantes y creyentes de la fe islámica que, ante una situación de extrema necesidad, optaron voluntaria y conscientemente por transgredir uno de los pilares esenciales que definen la pureza ritual del orden social que les era propio. De hecho, en el texto coránico se señala: «¿Qué razón tenéis para no comer de aquello sobre lo que se ha mencionado el nombre de Dios, habiéndoos Él detallado lo ilícito -salvo en caso de extrema necesidad-?» (Corán, VI: 119) ${ }^{12}$, advirtiendo que «Si alquien se ve compelido durante un hambre la comer de lo vedado], sin intención de pecar, ... Dios es indulgente y misericordioso» (Corán, V: 3).

En relación a esta cuestión, la documentación escrita y arqueológica ha permitido definir una situación en los espacios extramuros de Qurtuba durante los siglos XI y XII marcada por la inestabilidad y la inseguridad resultado de la ausencia (hasta finales del s. XII) de un poder consolidado y del consiguiente ambiente de agitación política y militar (BLANCO, 2014; LEÓN y BLANCO, 2010). Es muy posible que estos factores tuviesen consecuencias sobre los sistemas de producción ganadera y, particularmente, afectasen a los mecanismos de

12. La traducción del texto coránico citada es la de J. CORTÉS (2000). 
distribución y abastecimiento de los productos animales a los consumidores urbanos. Sin embargo, resulta igualmente necesario recordar el carácter aislado y de tipo marcadamente rural-o, en todo caso, periurbano- de la unidad de poblamiento que durante el s. XII se documenta en Cercadilla, por lo que parece poco probable que una eventual disrupción de los sistemas especializados de abastecimiento alimentario a la madina hubiese afectado de manera directa a este grupo poblacional. A pesar de ello, no debemos descartar que el contexto de inseguridad generalizada al que nos referimos repercutiese de alguna manera sobre las formas de gestión de los animales por parte de este grupo, quizá impidiendo la cría local orientada al autoconsumo con la que garantizar su supervivencia. En tal caso, quizá pudiera plantearse que el patrón de explotación y aprovechamiento de los animales que emerge de la muestra arqueofaunística examinada correspondiese a un evento puntual de consumo motivado por una situación de extrema necesidad que representase un riesgo real para la subsistencia de esta comunidad.

Con todo, y aun aceptando la posible existencia de una situación coyuntural de inestabilidad que hubiese supuesto un peligro manifiesto para la supervivencia a corto plazo del grupo, el levantamiento de la veda al consumo de suidos que se recoge en el Corán solo es aplicable en casos generalizados de escasez alimentaria. En la al-Muhallà, Ibn Hazm (m. 1064) incluye una referencia expresa a esta cuestión que, por el interés que presenta, se cita en extensión:

"The Muslim is not in a state of necessity such that it becomes permissible for him to eat the flesh of dead animals or swine as long as someone else, whether a Muslim or a dhimmi, has excess food. It is obligatory on the person having food to feed the one who is hungry, and that being the case, the hungry person is not compelled to resort to the flesh of dead animals or swine. If the person having excess food denies it to him, he has a right to fight for it. If he (the hungry person) is killed, the killer is guilty of murder and consequently subject to qisas (retaliation), while if he kills the denier of food he has dispatched him to the curse of Allah, as he denied him his right and was one of the rebellious» (IBN HAZM, cit. en AL-QARADAWI, 1999: 49).
Si otorgamos a la opinión del polígrafo cordobés la representatividad que, a nuestro juicio, merece, extraemos que la necesidad (la hambruna) elimina la prohibición específica del consumo de suidos siempre y cuando la magnitud del problema de subsistencia sea generalizada dado que, como sostienen FAROUK et al. (2015: 890), «necessity does not exist if the society possesses excess food».

Sin embargo, la información histórica y arqueológica disponible no permite plantear la existencia, al margen del clima de inestabilidad al que acabamos de hacer referencia, de una crisis de subsistencia de tal envergadura que implicase el desabastecimiento total de Qurtuba durante el s. XII. Pensamos, por lo tanto, que la transgresión alimentaria documentada en Cercadilla no responde a una decisión por parte del grupo residente en este espacio motivada por la necesidad ante una carestía generalizada de la que no encontramos eco en las fuentes disponibles. En consecuencia, y sin descartar una realidad poblacional definida por un marco productivo precario y formas de vida deficitarias, tras el comportamiento alimentario documentado probablemente se encuentran disposiciones de orden sociocultural que podrían ser indicio, en la línea de los argumentos de M. Benkheira, de un nivel de islamización social «de baja intensidad».

Ello nos conduce a la segunda de las hipótesis interpretativas que, a nuestro modo de ver, resultan más verosímiles para explicar el resultado que nos informa sobre un hábito de alimentación que incluye el consumo del producto harām por definición. La misma pasaría por asumir que nos encontramos ante residuos generados por un grupo social y culturalmente no islamizado. Siguiendo este planteamiento, de nuevo serían dos las opciones que se nos presentan.

De un lado, es posible que los individuos pertenecientes a este grupo se considerasen a sí mismos musulmanes, pero que, de manera consciente, pudiesen y quisiesen transgredir voluntariamente la norma dietética central sobre la que se asienta el sistema de pureza ritual del islam. Aunque desconocemos la 
regularidad en el tiempo de dicho comportamiento, en tal caso estaríamos ante un fenómeno similar al que ya hemos hecho alusión -si bien en referencia a otras cuestiones- consistente en el consumo, siempre puntual, de jabalí por parte de comunidades musulmanas eminentemente rurales. Una vez más, algunas referencias etnográficas al respecto ilustran bien este fenómeno.

Tras su visita a Sudán, a finales del s. XVIII, el explorador escocés J. BRUCE (1798: 371, cit. en LOBBAN, 1994: 64) relató que "hog's flesh is not sold in the market: but all the people of Sennar eat it publicly; men in office, who pretend to be Mohometans, eat theirs in secret». De manera más desapasionada se expresa B. GRANTHAM (1995: 74) cuando señala que, para los drusos de Siria, «religious law prohibits pig but some non-religious Druze do occasionally hunt wild boar on Mt. Hermon and consume the meat». Por último, a finales de los años 70, R. REDDING (2015: 334) presenció la caza y el consumo de jabalí en Irán, una práctica igualmente documentada, como ya hemos visto, en el Magreb (p. ej. BENKHEIRA, 1995, 1999; COON, 1931; MORENO, 2004; SIMOONS, 1994).

En la mayor parte de los casos por nosotros conocidos en que se documenta el consumo de jabalíes por parte de grupos musulmanes, de manera más o menos directa se enfatiza la laxitud religiosa de quienes practican dicho hábito de alimentación. Por lo tanto, la evidencia etnográfica sugiere que el jabalí, aun siendo consumido de manera ocasional -y, las más de las veces, subrepticiamente- por algunas comunidades musulmanes, no deja de ser considerado un animal ilícito en el contexto social islamizado en que se inscribe el campo cultural de acción de estas poblaciones (HART, 1976; ROSENBERGER, 1999). Por ello, el papel que desempeña este animal en el sistema de alimentación islámico en ningún caso puede equipararse al de las cabañas ganaderas (ancâm), proveedoras del único alimento que representa una fuente de baraka (BENKHEIRA, 2000). Dicho fenómeno es percibido «comme una rupture radicale avec la groupe», dado que «ceux qui en consomment son souvent des individus déjà marginalisés, au ban de la société, parfois dans une attitude de révolte» para los que este tipo de consumo representa «une façon de dire leur mépris, voire leur haine de la société» (BENKHEIRA, 1997: 267). Por consiguiente, «il n'est pas étonnant de rencontrer des Maghrébins que consomment du porc, dans le mesure où les sociétés maghrébines engendrent elles aussi leurs rebelles» (ibid., p. 268).

Podría pensarse que, en el caso de que los agentes generadores de los residuos examinados se considerasen a sí mismos como integrantes de la comunidad de creyentes que conforma la umma, el patrón de consumo documentado indicaría una islamización social «de baja intensidad». En la línea de lo mantenido por M. Benkheira (1997: 268), este tipo de consumo pudiera reflejar una práctica ritual blasfematoria que, en este contexto cultural, indicaría la salida de la esfera social islámica, puesto que «on ne peut manger du porc et continue à se dire musulman».

De otro lado, es igualmente razonable sugerir que los restos examinados fuesen acumulados por un grupo no musulmán que, dada la historia ocupacional de Cercadilla y el tipo de consumo documentado, podríamos interpretar como cristiano. En tal caso, el hábito de alimentación documentado no representaría una transgresión del código de alimentación de esta comunidad, pero sí de la normativa dietética de la formación social islámica en que se inserta.

Desde esta perspectiva también disponemos de evidencias etnográficas que, como la ya mencionada en relación a ciertas poblaciones armenias residentes en el Imperio otomano a inicios del s. XX (BÖKÖNYI, 1988), nos informan sobre la cría y consumo de suidos por parte de minorías cristianas residentes en el mundo islámico contemporáneo. Posiblemente el caso más conocido sea el de las comunidades coptas zabaleen que desde los años 40 se instalaron en El Cairo (OLDHAM, EL-HADIDI y TAMAA, 1987). También en Egipto, pero en una región rural, SIMOONS (1994: 340) señala la presencia de pequeñas piaras de 
cerdos en zonas donde las poblaciones coptas son numerosas, como en la ciudad de Al Bayadiyyah, donde «free-ranging pigs are owned by many families, and move unattended along the streets».

En Cercadilla, la documentación arqueozoológica expuesta anteriormente revela un patrón diacrónico de enorme interés que indica el abandono de la cría de porcino entre época emiral (ss. VIII-IX) y califal (ss. X-XI), lo que interpretamos como el resultado de la islamización social y cultural de esta población con la consecuente adopción de los hábitos de alimentación propios del modelo social islámico (GARCÍA GARCÍA, 2019). Disponemos igualmente de referencias documentales que nos informan sobre el mismo fenómeno. Quizá la más reveladora en este sentido sea la que recoge el supuesto diálogo entre un obispo cordobés de nombre Juan y un monje lorenés enviado por el emperador Otón la Qurtuba a mediados del s.X. Siguiendo la traducción de PAZ Y MELIÁ (1931), el lorenés recriminó a su correligionario la abstinencia por parte de la comunidad cristiana cordobesa del consumo de «ciertos alimentos que Dios creó para que con acción de gracias participasen de ellos los fieles», una actitud que el obispo mozárabe justifica por la necesidad del ambiente social en que habitan, añadiendo que «así lo hemos recibido de nuestros mayores por una larga tradición». Tanto SIMONET (1983) Como GARCÍA SANJUÁN (2009) asumen que la mención a esos alimentos se refiere específicamente al cerdo, lo que es interpretado por el segundo de estos autores como una manifestación más, entre otras que documenta, del desapego y rechazo de los cristianos extrapeninsulares hacia los de al-Andalus debido a la aculturación experimentada por los segundos.

Así pues, parece que, al menos desde el s. X, parte de la comunidad cristiana cordobesa se encontraba plenamente integrada en el marco social islamizado en lo referente a diversos aspectos entre los que destacan para nuestros intereses, amén de otros como los lingüísticos o jurídicos (AILLET, 2010, 2011; GARCÍA SANJUÁN, 2009), los alimentarios. Sin embargo, sobre la base del conjunto de datos disponibles, no creemos que ello sea óbice para considerar como la hipótesis interpretativa más verosímil la posible identidad cristiana del grupo generador de este basurero debido, además de las ya señaladas, a una evidencia adicional referida en este trabajo como es la valva perforada de vieira. Dado el carácter particular, ciertamente diferenciado con respecto al basurero al que hasta ahora venimos atendiendo, y al origen contextual del artefacto en cuestión, abordaremos la discusión de las implicaciones socioculturales que presenta en la siguiente sección.

\subsection{La valva derecha perforada de vieira Pecten maximus}

Entre el material arqueofaunístico recuperado de la fase tardoandalusí de Cercadilla destaca una valva derecha de vieira atribuida específicamente a Pecten maximus, que presentaba dos perforaciones en la zona del borde del umbo practicadas con toda probabilidad para adaptarla a su uso como colgante.

La presencia de este bivalvo no es del todo infrecuente en contextos arqueológicos andalusíes (GARCíA GARCÍA, 2019). Sin embargo, el derivado de Cercadilla se trata del único caso de los registrados en al-Andalus en que, hasta donde nosotros sabemos, se documentan perforaciones sobre una valva derecha que indican un uso ornamental de la pieza. Ambas características permiten, a nuestro juicio, atribuir al ejemplar un significado cultural y simbólico que remite a un tipo circunscrito y específico de religiosidad marcadamente cristiana como es la peregrinación Jacobea (GIROD, 2001; KÖSTER, 1985). La estrecha semejanza que presenta este resto con otros similares identificados, entre otros casos, en Liguria (GIROD, 2001), York (GILCHRIST, 2008), Winchester (ROFFEY et al., 2017), el Castro de los Judíos (GONZÁLEZ et al., 2010) (Fig. 9) o en el Mediterráneo oriental (KTALAV, 2016), contextos todos ellos asociados, directa o indirectamente, al mundo cristiano medieval europeo, no hace sino reforzar su interpretación como una concha de peregrino. 


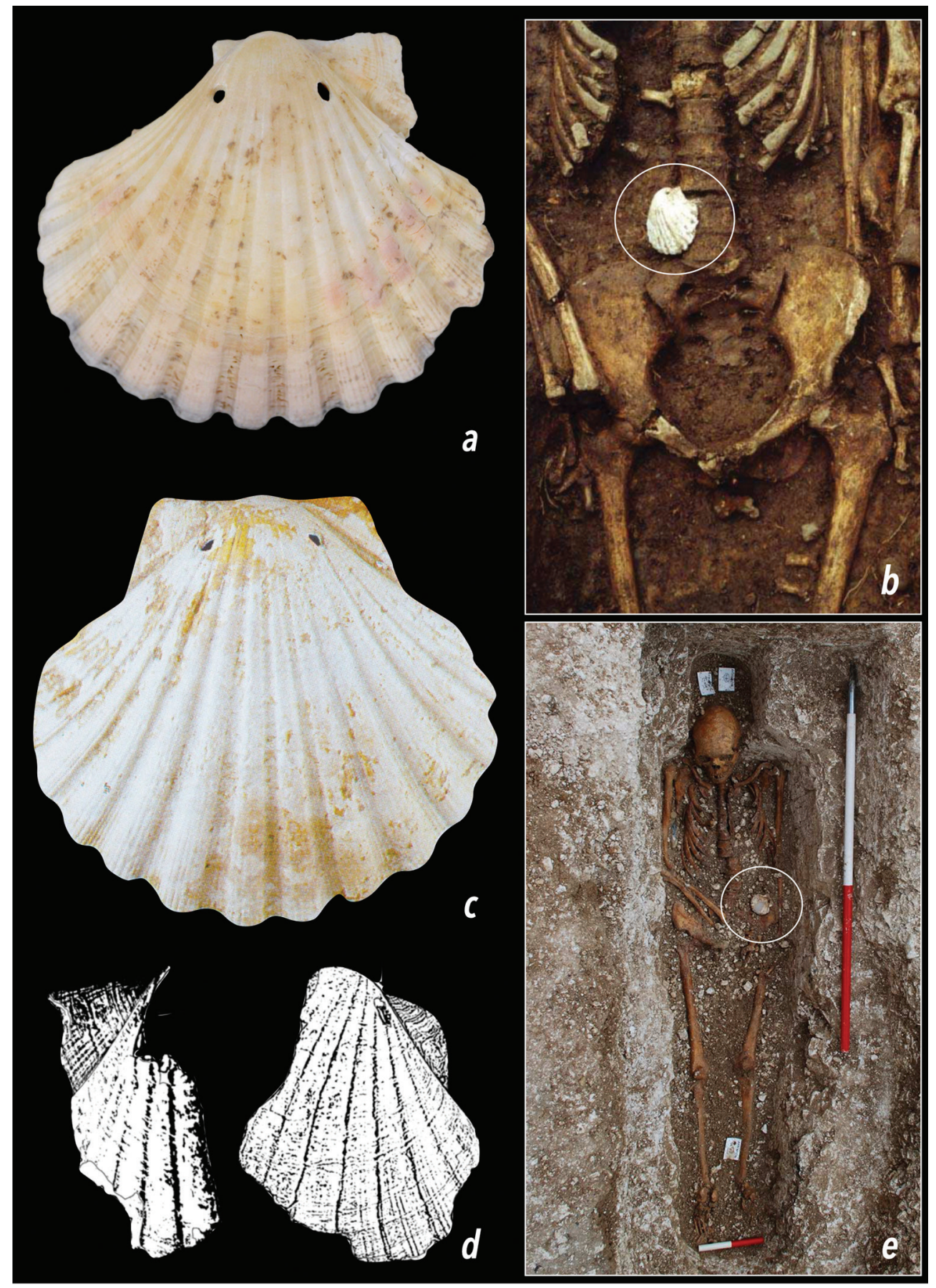

Fig. 9. Ejemplos de valvas derechas de Pecten maximus perforadas similares a la de Cercadilla (a): b) St Helen's Fishergate, York (modificado a partir de: GILCHRIST, 2008: Fig. 2); c) Albenga, Santa Maria in Fontibus, Liguria (GIROD, 2001: Fig. 27); d) Castro de los Judios, León (GONZÁLEZ et alii, 2010: Fig. 3); e) Leprosarium of St Mary Magdalen, Winchester (modificado a partir de ROFFEY et alii, 2017: Fig. 1).

A este respecto, no nos parece casual que sea precisamente en el s. XII cuando, por primera vez, aparece documentada la utilización de valvas derechas de P. maximus -las únicas utilizadas en la simbología Jacobea (GIROD, 2001)- como insignia de los peregrinos compostelanos, coincidiendo con el momento de eclosión de la ruta a Santiago. Esta primera atestiguación documental que nos informa sobre la costumbre de los peregrinos de coser las conchas en sus capas, sombreros y escarcelas está recogida en el sermón XVII del Libro 
I del Liber Sancti lacobi (c. 1140) (ANGUITA, 1996), en donde se señala que: «[...] las cosen los peregrinos que vuelven de Santiago en sus capas en honor del apóstol y en memoria suya, llevándolas con gran alegría a sus casas como señal de tan alegre viaje» (VÁZQUEZ DE PARGA, LACARRA y URÍA, 1948: 130, extraído del Liber Sancti lacobi).

Creemos asimismo relevante volver a enfatizar el hecho de que el contexto de origen del resto que nos ocupa no sea un basurero, en cuyo caso debiéramos cuestionar esta posible atribución funcional o simbólica al aparecer descartado junto a otros residuos. Bien al contrario, al provenir de un suelo de ocupación de un espacio habitacional, asumimos que este artefacto tuvo un papel utilitario (decorativo, profiláctico, apotropaico, ...) en el contexto sistémico (sensu SCHIFFER, 1972) de los residentes de este espacio cordobés durante el s. XII.

Siguiendo a J. M. ANGUITA (1996: 52), la concha de Pecten maximus se asocia en el mundo cristiano medieval a varios valores, entre los que destacan el de distintivo -emblema que marca al devoto peregrino de Santiago-, amuleto debido al carácter protector aprotropaico-, adorno -un objeto estético que llenaba de orgullo al portador-y prueba que acreditaba la consecución de la peregrinación al santuario de Santiago en el «Fin de la Tierra». Asimismo, en la literatura en torno a este tipo de objetos, estos aparecen dotados de poder sobrenatural vinculado con sanaciones o actuaciones milagrosas que abundan en el valor mágico-propiciatorio de la concha por su relación con el Apóstol Santiago (ibid.).

Este conjunto de valores, cualidades y poderes atribuidos a las conchas derechas de Pecten maximus determinó el desarrollo de un comercio destinado a su venta en el mercado de la plaza de la catedral de Compostela, un negocio considerado suficientemente lucrativo como para que, en el año 1200, la Iglesia reivindicase su monopolio frente a los concheiros y el derecho exclusivo para autorizar su venta (VÁZQUEZ DE PARGA et al., 1948: 132). La importancia que debió tener el negocio de la venta de estos «souvenirs de pèlerinage typiquement médiévales» (KÖSTER, 1985: 85) se refleja igualmente en el empeño que, desde las mismas fechas, se pone por atajar y perseguir las falsificaciones, prohibiendo a los peregrinos comprar o llevar conchas que no se hubiesen elaborado en Compostela, así como fabricarlas fuera de la ciudad (VÁZQUEZ DE PARGA et al., 1948: 133-4).

Consideramos que esclarecer si la concha que nos ocupa es una falsificación o si, efectivamente, se trata de un objeto adquirido en Compostela, es un problema menor -imposible de resolver con la información actualmente a disposición- ya que, sea como fuere, su simple presencia en el contexto habitacional referido presenta un indudable interés por el horizonte cultural y religioso al que, en nuestra opinión, remite de manera directa.

\section{CONCLUSIONES}

En este trabajo se ha presentado y discutido un conjunto de informaciones derivadas del estudio del material arqueofaunístico recuperado de una unidad de poblamiento que se desarrolló en un espacio periférico y relativamente aislado de madinat Qurtuba durante el s. XII. La evidencia recabada revela unos patrones de alimentación y un consumo de cultura material que permite sugerir como hipótesis interpretativa la posible identidad cristiana de los actores responsables de la acumulación de los restos examinados, en cuyo caso cabría asumir su pertenencia a la comunidad cristiana dimmí o mozárabe. El examen de un conjunto de restos animales que conformaban parte de un basurero acumulado en el interior de una estructura negativa y que pueden interpretarse sin demasiado riesgo como residuos de alimentación, reveló la presencia predominante del grupo taxonómico de los suidos. Sobre la base de las reflexiones de orden teórico expuestas a lo largo de las páginas precedentes, y dejando al margen la identificación taxonómica específica de estos restos -es decir, si pertenecieron a cerdo doméstico o a jabalí salvaje-, este resultado testimonia un comportamiento de gran significación en términos socioculturales, 
dado que supone una transgresión evidente de la normativa dietética propia de la ortopraxis del islam medieval. Es posible que la particular distribución anatómica de la muestra guarde igualmente relación con este hábito de alimentación singular, lo que explicaría la adopción de estrategias de gestión y aprovechamiento «especiales».

Por su parte, la identificación de una valva derecha perforada de vieira Pecten maximus en un nivel de frecuentación supone un hallazgo de gran interés que nos informa sobre el consumo de un tipo de cultura material vinculado al horizonte cultural y simbólico cristiano. La ausencia de este tipo de artefactos en otros contextos andalusíes y la similitud que guarda con otros aparecidos en el ámbito cristiano medieval europeo no hacen sino reforzar la atribución de este resto como una concha de peregrino asociada, si quiera en términos meramente simbólicos, a la ruta Jacobea.

Los resultados y las interpretaciones realizadas reflejan el potencial informativo del material arqueofaunístico, como registro de las formas de relación de las sociedades pretéritas con los animales y sus productos derivados, para arrojar luz sobre la identidad sociocultural y etnorreligiosa de los actores sociales, particularmente cuando la presencia de ciertos grupos o comunidades minoritarias aparece silenciada en la documentación escrita. En esta ocasión, los datos arqueozoológicos nos han permitido detectar la presencia de un grupo que estimamos razonable identificar como cristiano en un contexto histórico, el de la Qurtuba del s. XII, en que estas comunidades desaparecen de las principales fuentes escritas. Como apunta A. HARRISON (2009, 2012), esta ausencia documental ha sido tomada como argumento ex silentio sobre el que se ha construido una imagen del s. XII marcada por la inexistencia -resultado de la conversión, persecución o la emigración- de dichas comunidades en al-Andalus. La arqueología, una vez más, nos permite conocer y visibilizar lo que otras fuentes históricas esconden y, de esto modo, contribuir a la generación de imágenes del pasado más ricas y complejas.

\section{AGRADECIMIENTOS}

Agradecemos a E. González (Universidad de León) la ayuda con la identificación taxonómica de la valva de vieira, a J. M. Anguita (Universidad de Santiago) que compartiese con nosotros sus reflexiones sobre la posible interpretación de este artefacto y a G. García-Contreras (Universidad de Granada) la lectura del manuscrito y la aportación de interesantes comentarios que contribuyeron sustancialmente a su mejora.

\section{BIBLIOGRAFÍA}

ACIÉN ALMANSA, M (1997) Entre el feudalismo y el islam. 'Umar b. Hafsūun en los historiadores, en las fuentes y en la historia, 2. ${ }^{a}$ ed. Jaén: Universidad de Jaén.

ACIÉN ALMANSA, M y VALLEJO TRIANO, A (1998) «Urbanismo y Estado islámico: de Corduba a Qurtuba-Madinat al-Zahrā'», en P. Cressier y M. García-Arenal (Eds.), Genèse de la ville islamique en al-Andalus et au\{Maghreb occidental, pp. 107-136. Madrid: Casa de Velázquez, Consejo Superior de Investigaciones Científicas.

AILLET, C (2010) Les «Mozarabes»: Christianisme, islamisation et arabisation en péninsule ibérique (IXe-XIle siècles). Madrid: Casa de velázquez.

AILLET, C (2011) «Islamisation et évolution du peuplement chrétien en al-Andalus (VIIle-XIle siècle)», en D. Valérian (Ed.), Islamisation et arabisation de l'Occident musulman médiéval, pp. 151-192. París: Publications de la Sorbonne, Bibliothéque Historique des Pays d'Islam.

ALBARELLA, U (2002) «'Size matters': how and why biometry is still important in zooarchaeology», en K. Dobney y P. O'Connor (Eds.), Bones and the Man. Studies in honour of Don Brothwell, pp. 51-62. Oxford: Oxbow Books.

ALBARELLA, U (2015) «Defining bone movement in archaeological stratigraphy: a plea for clarity», Archaeol Anthrop Sci, 8(2), 353-358. DOI: 10.1007/s12520-015-0269-9.

Al-QARADAWI, Y (1999) The lawful and the prohibited in Islam. Plainfield: American Trust Publications.

ANGUITA, José Ma (1996) «La concha jacobea ('vieira') en el Liber Sancti lacobi (Codex Calixtinus)». lacobvs. Revista de Estudios Jacobeos y Medievales, 1, 47-54.

APPADURAI, Arjun (1981) "Gastro-politics in Hindu South Asia», Am Ethnol, 8(3), 494-511. DOl: https://doi.org/10.1525/ ae.1981.8.3.02a00050.

ARCAS CAMPOY, María (1996) «Los alimentos en el derecho māliki», Boletín de la Asociación Española de Orientalistas, Año XXXII, 111-119. 
ARCAS CAMPOY, María (2016) «El criterio de los juristas malikíes sobre los alimentos y las bebidas de los dimmíes: entre la teoría y la práctica», en A. Echevarría, J. P. Monferrer y P. Tolan (Eds.), Law and religious minorities in medieval societies: between theory and praxis, pp. 85-100. Turnhout: Brepols.

ASAD, Tel (1986) The idea of an anthropology of islam. Washington DC: Center for Contemporary Arab Studies.

ATALAY, Sonya y HASTORF, Christine (2006) «Food, meals and daily activities: food habitus at Neolithic Çatalhöyük», Am Antiq, 71(2), 283-319. DOI: 10.2307/40035906.

BARTH, Fredrik (1969) Ethnic groups and boundaries: the social organisation of cultural difference. Bergen: Universitets Forlaget.

BENDER, B; HAMILTON, S. y TILLEY, C. (1997) «Leskernick; Stone Worlds; Alternaive Narratives; Nested Landscapes», Proc Prehist Soci, 63, 147-178.

BENKHEIRA, Mohammed (1995) «La Nourriture carnée comme frontière rituelle», Arch Sci Soc Relig, 92, 67-88.

BENKHEIRA, Mohammed (1997) "Alimentation, altérité et socialité: remarques sur les tabous alimentaires coraniques», European Journal of Sociology, 38(2), 237-287.

BENKHEIRA, Mohammed (1999) «Lier et séparer. Les fonctions rituelles de la viande dans le monde islamisé», L'Homme, 152, 89-114.

BENKHEIRA, Mohammed (2000) Islâm et interdits alimentaires. Juguler l'animalitè. París: Presses Universitaires de France.

BENKHEIRA, Mohammed (2002) «Tabou du porc et identité en Islam», en M. Bruegel y B. Laurioux (Eds.), Histoire et identités alimentaires en Europe, pp. 37-52. París: Hachettes littératures.

BENKHEIRA, Mohammed (2006) "Quelques interprétations anthropologiques du tabou du porc en islâm», en B. Lion, Brigitte y C. Michel (Eds.), De la domestication au tabou: le cas des suidés dans le Proche-Orient ancien, pp. 233-244. París: De Boccard.

BENTLEY, G. Carter (1987) «Ethnicity and practice», Comparative Studies in Society and History, 29:1, pp. 24-55. DOI: https://doi. org/10.1017/S001041750001433X.

BLANCO GUZMÁN, Rafael (2014) La arquitectura doméstica tardoislámica de Qurtuba (ss. XI-XIII). Córdoba: Universidad de Córdoba.

BÖKÖNYI, Sandor (1988) History of domestic mammals in Central and Eastern Europe. Budapest: Akadémiai Kiadó.

BOURDIEU, Pierre (1980) Le sens pratique. París: Les Éditions de Minuit.

BRUCE, James (1798) An interesting narrative of the travels of James Bruce into Abyssinia to discover the source of the Nile (American abridged version). Boston: Etheridge.

CARMONA, Alfonso (2007) «Una década (1997-2007) de trabajos sobre textos religiosos y jurídicos de al-Andalus», Medievalismo, 17, 291-312.

COLYER, F. (1936) Variations and diseases of the teeth of animals. Londres: John Bale, Sons and Danielson.
COOK, Michael (1986) «Early Islamic dietary law», Jerusalem Studies in Arabic and Islam, 7, 217-277.

COON, C. (1931) Tribes of the Rif. Cambridge, MA.: Peabody Museum of Harvard University.

CORTÉS, Julio (2000) El Corán. Barcelona: Herder.

DAVIS, Simon J.M. (1996) «Measurements of a group of adult female Shetland sheep skeletons from a single flock: a baseline for zooarchaeologists», J Archaeol Sci, 23(4), 593-612. DOI: 10.1006/ jasc.1996.0056.

DOBNEY, Keith y ERVYNCK, Anton (1998) «A protocol for recording enamel hypoplasia on archaeological pig teeth», Int J Osteoarchaeol, 8(4), 263-274.

DOBNEY, Keith; ERVYNCK, Anton; ALBARELLA, Umberto y ROWLEY-CONWY, Peter (2004) «The chronology and frequency of a stress marker (linear enamel hypoplasia) in recent and archaeological populations of Sus scrofa in north-west Europe, and the effects of early domestication», J Zool, 264, 197-208. DOl: https://doi.org/10.1002/(SICI)1099-1212(199807/08)8:4<263::AIDOA427>3.0.CO;2-P.

DOBNEY, Keith; ERVYNCK, Anton; ALBARELLA, Umberto y ROWLEY-CONWY, Peter (2007) «The transition from wild boar to domestic pig in Eurasia, illustrated by a tooth developmental defect and biometrical data», en U. Albarella (Ed.), Pigs and Humans. 10,000 years of interaction, pp. 57-82. Oxford: Oxford University Press.

DOUGLAS, Mary (1984) Food in the social order. Nueva York: Russell Sage Foundation.

FAROUK, M. M.; REGENSTEIN, J. M.; PIRIE, M. R.; NAJM, R; BEKHIT, A. E. D. y KNOWLES, S. O. (2015) «Spiritual aspects of meat and nutritional security: Perspectives and responsibilities of the Abrahamic faiths», Food Res Int, 76(4), 882-895. DOI: https://doi.org/10.1016/j. foodres.2015.05.028.

FERNÁNDEZ FÉLIX, Ana (2003) Cuestiones legales del islam temprano: la 'Utbiya y el proceso de formación de la sociedad islámica andalusí. Madrid: Consejo Superior de Investigaciones Científicas.

FIDDES, Nick (1991) Meat, a natural symbol. Londres: Routledge.

FIERRO, Maribel (1997) «Christian success and Muslim fear in Andalusi writings during the Almoravid and Almohad periods», en U. Rubin y D. J. Wasserstein (Eds.), Israel Oriental Studies XVII. Dhimmis and others: Jews and Christians and the World of Classical Islam, pp. 155-178. Tel Aviv: Eisenbrauns.

FIERRO, Maribel (2004) «La política religiosa de 'Abd al-Rahmān III (r. 300/912-350/961)», Al-Qanțara, XXV(1), 119-156.

FISCHLER, Claude (1988) «Food, self and identity», Soc Sci Inf, 27, pp. 275-292.

FUERTES SANTOS, Ma del C. (2006) «Córdoba durante el siglo XII. El abandono y ruina de los arrabales occidentales y su reconversión en espacio agrícola e industrial a través de las excavaciones de Cercadilla», en D. Vaquerizo y J. Murillo (Eds.), El concepto de 10 provincial en el mundo antiguo. Homenaje a la profesora Pilar León Alonso, pp. 439-462. Córdoba: Universidad de Córdoba.

FUERTES SANTOS, Ma del C. (2009) «El siglo XII en Cercadilla a través de los materiales cerámicos. Avance de resultados», en J. Zozaya, 
M. Retuerce, M.Á. Hervás y A. de Juan (Eds.), Actas del VIII Congreso Internacional de Cerámica Medieval en el Mediterráneo (Ciudad Real-Almagro, del 27 de febrero al 3 de marzo de 2006), pp. 327-337. Ciudad Real: Asociación Española de Arqueología Medieval.

FUERTES SANTOS, Ma del C. (2010) La cerámica medieval de Cercadilla, Córdoba. Tipología, decoración y función. Sevilla: Junta de Andalucía.

FUERTES SANTOS, Ma del C. (2018) «Una tumba insólita de la necrópolis mozárabe de Cercadilla, Córdoba», en E. Cerrato y D. Asensio (Eds.), Nasara, extranjeros en su tierra. Estudios sobre cultura mozárabe y catálogo de la exposición, pp. 33-46. Córdoba: Cabildo Catedral de Córdoba.

FUERTES SANTOS, Ma del C. e HIDALGO PRIETO, Rafael (2005) Guía Arqueológica de Cercadilla. Córdoba. Sevilla: Junta de Andalucía, Consejería de Cultura.

FUERTES SANTOS, Ma del C. e HIDALGO PRIETO, Rafael (2010) «La transformación del paisaje del área noroccidental cordobesa y de palacio imperial de Maximiano tras la caída de la Tetrarquía», en A. García, R. Izquierdo, L. Olmo y D. Peris (Eds.), Espacios urbanos en el occidente mediterráneo (ss. VI-VIII), pp. 165-172.Toledo: Toletvm Visigodo.

FUERTES SANTOS, Mª del Camino; RODERO PÉREZ, Santiago y ARIZA RODRÍGUEZ, Javier (2007) «Nuevos datos urbanísticos en el área de la puerta del Palatium de Córdoba», Romula, 6, 173-210.

GARCÍA GARCÍA, Marcos (2019) Explotación y consumo de los animales en el sudeste de la península ibérica durante la Alta Edad Media (siglos VII-XII) perspectivas históricas y arqueozoológicas. Granada: Universidad de Granada. DOI: http://hdl.handle. net/10481/55386

GARCÍA SÁNCHEZ, Expiración (1992) Abū Marwān 'Abd al-Malik B. Zuhr (m. 557/1162). Kitāb al-Agdiya (Tratado de los alimentos). Madrid: Consejo Superior de Investigaciones Científicas/Instituto de Cooperación con el Mundo Árabe.

GARCÍA SANJUÁN, Alejandro (2002) «El consumo de alimentos de los dimmíes en el islam medieval: prescripciones jurídicas y prác tica social», Historia. Instituciones. Documentos, 29, 109-146.

GARCÍA SANJUÁN, Alejandro (2004) «Declive y extinción de la minoría cristiana en la Sevilla andalusí (ss. XI-XII)», Historia. Instituciones. Documentos, 31, 269-286.

GARCÍA SANJUÁN, Alejandro (2009) «El fin de las comunidades cristianas de al-Andalus (siglos XI-XII) factores de una evolución», en XI Congreso de Estudios Medievales. Cristianos y musulmanes en la península Ibérica: la guerra, la frontera y la convivencia (León, 23-26 octubre 2007), pp. 259-287. León: Fundación Sánchez Albornoz.

GILCHRIST, Roberta (2008) «Magic for the dead? The archaeology of magic in later medieval burials», Medieval Archaeology, 52, 119159. DOl: https://doi.org/10.1179/174581708×335468.

GIROD, A. (2001) «Le conchiglie. Studio malacologico», en F. Bulgarelli, A. Gardini, y P. Melli (Eds.), Archeologica dei pellegrinaggi in Liguria, pp. 44-49. Savona: Sopraintendenza Archeologica della Liguria.

GONZÁLEZ GÓMEZ DE AGÜERO, Eduardo; BEJEGA GARCÍA, Víctor FERNÁNDEZ RODRÍGUEZ, Carlos y ÁLVAREZ GARCÍA, Juan C. (2010) «La presencia de moluscos en un asentamiento judío durante la
Edad Media: El Castro de los Judíos (Puente Castro, León)», Férvedes, 6, 159-168.

GRANTHAM, Bill (1995) «Dinner in Buqata: the symbolic nature of food animals and meal sharing in a Druze village», en K. Ryan y P. Crabtree (Eds.), The symbolic role of animals in archaeology. MASCA Research papers in Science and Archaeology, 12, pp. 73-78. Filadelfia: MASCA, University of Pennsylvania Museum of Archaeology and Anthropology.

GRAU SOLOGESTOA, Idoia (2015) The zooarchaeology of medieval Alava in its Iberian context. Oxford: BAR Int. Series 2769.

HALL, S. (1996) «Introduction: who needs 'identity'?», en S. Hall y P. Du Gay (Eds.), Questions of cultural identity, pp. 1-17. Londres: Sage.

HARRISON, Alwyn (2009) Andalusi Christianity: the survival of indigenous Christian communities, tesis doctoral. Exeter: University of Exeter.

HARRISON, Alwyn (2012) «Behind the curve: Bulliet and conversion to Islam in al-Andalus revisited», Al-Masāq, 24(1), 35-51.

HART, David M. (1976) The Aith Waryaghar of the Moroccan Rif. An ethnography and History. Tucson: University of Arizona Press.

HIDALGO PRIETO, Rafael (1996) Espacio público y espacio privado en el conjunto palatino de Cercadilla (Córdoba) el aula central y las termas. Sevilla: Consejería de Cultura.

HIDALGO PRIETO, Rafael (2002) «De edificio imperial a complejo de culto: la ocupación cristiana del palacio de Cercadilla», en D. Vaquerizo (Ed.), Espacios y usos funerarios en el Occidente romano (Vol. II), pp. 343-372. Córdoba: Seminario de Arqueología (UCO).

HIDALGO PRIETO, Rafael (2004) «El palatium», en X. Dupré (Ed.), Las capitales provinciales de Hispania. 1, Córdoba, Colonia Patricia Corduba, pp. 95-104. Roma: L'Erma di Bretschneider.

HIDALGO PRIETO, Rafael (2014) «iFue Cercadilla una villa? El problema de la función del complejo de Cercadilla en Corduba», Archivo Español de Arqueología, 87, 217-241.

HIDALGO PRIETO, Rafael (2016) «El complejo monumental de Cercadilla: las transformaciones cristianas», en O. Brandty G. Castiglia (Eds.), Acta XVI Congressvs Internationalis Archaeologiae Christianae. Costantino e i costantinidi. L'innovazione costantiniana, le sue radici e i suoi sviluppi, pp. 523-550. Ciudad del Vaticano: Pontificio Istituto di Archaeologia Cristiana.

HIDALGO PRIETO, Rafael y VENTURA VILLANUEVA, Ángel (1994) «Sobre la cronología e interpretación del palacio de Cercadilla en Corduba», Chrion, 24, 221-240.

HODGSON, Marshall G. S. (1974) The venture of Islam. Conscience and History in a world civilization. The classical age of islam (Vol. I). Chicago: The University of Chicago Press.

INSKIP, Sarah (2013) Islam in Iberia or Iberian Islam: Sociobioarchaeology and the analysis of emerging islamic identity in early medieval Iberia, tesis doctoral. Southampton: University of Southampton.

INSOLL, Tim (1999) The archaeology of Islam. Oxford: Blackwell.

INSOLL, Tim (2004) Archaeology, ritual, religion. Londres: Routledge.

INSOLL, Tim (2007) The archaeology of identities. Londres: Routledge. 
JONES, Siân (1997) The archaeology of ethnicity. Constructing identities in the past and present. Londres: Routledge.

KÖSTER, Kurt (1985) «Les coquilles et enseignes de pèlerinage de Saint-Jacques de Compostela et les routes de Saint-Jacq», en Santiago de Compostela: 1000 ans de pèlerinage, pp. 85-95. Gand: Centrum Voor Kunst En Cultuur.

KRUK, Remke (2019) «The saddest beast? Notes on the pig in Arabic culture», Quaderni di Studi Arabi (Nuova Serie), 14, 243-261.

KTALAV, Inbar (2016) «There and back again: a tale of a pilgrim badge during the Crusader Period», en N. Maron, R. Yeshurun, L. Wissbrod y G. Bar-Oz (Eds.), Bones and Identity. Zooarchaeological Approaches to Reconstructing Social and Cultural Landscapes in Southwest Asia, pp. 323-338. Oxford: Oxbow.

LEÓN MUÑOZ, Alberto y BLANCO MUÑOZ, Rafael (2010) «La fitna y sus consecuencias. La revitalización urbana de Córdoba en época almohade», en D. Vaquerizo y J.F. Murillo (Eds.), El anfiteatro romano de Córdoba y su entorno urbano. Análisis arqueológico (ss. I-XIII d.C.), pp. 699-726. Córdoba: Servicio de Publicaciones de la Universidad de Córdoba.

LEÓN MUÑOZ, Alberto y MURILLO REDONDO, Juan F. (2014) "Advances in Research on Islamic Cordoba», J Islam Archaeol, 1(1), 5-35. DOI: 10.1558/jia.v1i1.5

LEVY-RUBIN, Milka (2011) Non-muslims in the Early Islamic Empire. From surrender to coexistence. Cambridge: Cambridge University Press.

LEVY-RUBIN, Milka (2013) «The Pact of 'Umar», en G. Böwering, P. Crone, W. Kadi, D. Steward, M. Zaman y M. Mirza (Eds.), The Princeton Encyclopedia of Islamic political thought. Princeton: Princeton University Press.

LOBBAN, Richard A. (1994) «Pigs and their prohibition», Int. J. Middle East Stud., 26(1), 57-75. DOI: 10.1017/S0020743800059766.

LUCY, Sam (2005) «Ethnic and cultural identities», en M. DíazAndreu, S. Lucy, B. Staša y D. Edwards (Eds.), The archaeology of identity, pp. 86-109. Abingdon: Routledge.

MANZANO MORENO, Eduardo (1994) «Nota crítica: Entre el Feudalismo y el Islam. 'Umar ibn Hafsun en los historiadores, en las fuentes y en la historia, de M. Acién Almansa», Hispania, 54(3), 1139-1144.

MARÍN NIÑO, Manuela (2004) «Espacios religiosos en las sociedades islámicas clásicas: una aproximación», Revista de ciencias de las religiones. Anejos, 10, 93-102.

MARTÍNEZ SÁNCHEZ, Rafael (2017) «Animales en Córdoba durante el primer siglo andalusí. Revisando los basureros del arrabal de Saqunda», en S. Villar y M. García (Eds.), Ganadería y Arqueología medieval, pp. 17-56. Granada: Alhulia.

MEADOW, Richard H. (1999) «The use of size index scaling techniques for research on archaeozoological collections from the Middle East», en C. Becker, H. Manhart, J. Peters y J. Schibler (Eds.), Historia Animalium ex Ossibus. Festschrift für Angela von den Driesch, pp. 285-300. Rahden: Verlag Marie Leidorf GmbH.

MORENO GARCÍA, Marta (2004) «Hunting practices and consumption patterns in rural communities in the Rif mountains (Morocco). Some ethno-zoological notes», en Sh. J. O'Day, W. Van Neer y A. Ervynck (Eds.), Behaviour behind bones. The zooarchaeology of ritual, religion, status and identity, pp. 327-334. Oxford: Oxbow Books.

O'CONNOR, Terry P. (1992) «Provisioning urban communities: A topic in search of a model», Anthropozoologica, 16, 101-106.

O'CONNOR, Terry P. (2000) The archaeology of animal bones. Strout: Sutton Publishing.

O'CONNOR, Terry P. (2003) The analysis of urban animal bones assemblages: a handbook for archaeologists. York: York Archaeological Trust.

O'CONNOR, Terry P. (2006) «Vertebrate demography by numbers: age, sex, and zooarchaeological practice», en D. Ruscillo (Ed.), Recent advances in ageing and sexing animal bones, pp. 1-8. Oxford: Oxbow Books.

OLDHAM, Linda; EL-HADIDI, Hajir y TAMAA, Hussein (1987) «Informal communities in Cairo: the basis of a typology», Cairo Papers in Social Science (whole issue), 10(4).

ORTIZ RAMÍREZ, Laura (2003) «Los Mozárabes de Córdoba. Una aproximación preliminar a la necrópolis de Cercadilla», Arte, Arqueología e Historia, pp. 79-84.

PAZ Y MELIÁ, Antonio (1931) «La embajada del Emperador de Alemania Otón I al califal de Córdoba Abderrahmán III», Boletín de la Academia de Ciencias, Bellas Artes y Nobles Artes de Córdoba, 33, 123-150.

REDDING, Richard W. (1991) «The role of the pig in the subsistence system of Ancient Egypt: a parable of the potential of faunal data», en P. Crabtree y K. Ryan (Eds.), Animal use and culture change, pp. 20-30. Filadelfia: University of Pennsylvania, Museum of Archaeology and Anthropology Research Papers in Science and Archaeology (MASCA, 8).

REDDING, Richard W. (2015) «The pig and the chicken in the Middle East: modeling human subsistence behavior in the archaeological record using historical and animal husbandry data», J Archaeol Res, 23, 325-368. DOI: https://doi.org/10.1007/ s10814-015-9083-2.

ROFFEY, Simon; TUCKER, Katie; FILIPEK-OGDEN, Kori; MONTGOMERY, Janet; CAMERON, Jamie; O'CONNELL, Tamsin; EVANS, Jane; MARTER, Phil y TAYLOR, G. Michael (2017) «Investigation of a medieval pilgrim burial excavated from the Leprosarium of St Mary Magdalen Winchester, UK», PLOS Neglected Tropical Diseases, 11(1). DOl: https://doi.org/10.1371/journal.pntd.0005186.

ROSENBERGER, Bernard (1999) «Arab cuisine and its contribution to European culture», en J.L. Flandrin y M. Montanari (Eds.), Food: a culinary history from Antiquity to the present, pp. 207-223. Nueva York: Columbia University Press.

ROZIN, Paul (1987) «Psychobiological perspectives on food preferences and avoidances», en M. Harris y E.B. Ross (Eds.), Food and evolution, pp. 181-205. Filadelfia: Temple University Press.

SAFRAN, Janina (2003) «Rules of purity and confessional boundaries: maliki debates about the pollution of the christian», Hist Relig, 42(3), 197-212

SALINAS, Elena (2012) «La cerámica islámica de madinat Qurțuba de 1031 a 1236: cronotipología y centros de producción», tesis doctoral. Córdoba: Universidad de Córdoba. 
SARNAT, H. y MOSS, S. J. (1985) "Diagnosis of enamel defects», N Y State Dent J, 51(2), 103-106. DOI: 10.1002/j.1875-595x.1997.tb00783.x.

SCHIFFER, Michael (1972) «Archaeological context and systemic context», Am Antiq, 37, 156-165. DOI: 10.2307/278203.

SCHOLLIERS, Peter (2001) «Meals, food narratives and sentiments of belonging in past and present», en P. Scholliers (Ed.), Food, drink and identity: cooking, eating and drinking in Europe since the Middle Ages, pp. 3-22. Oxford: Berg.

SHERRATT, Andrew (1995) «Alcohol and its alternatives: symbol and substance in pre-industrial cultures», en J. Goodman, P. Lovejoy y A. Sherratt (Eds.), Consuming habits: Drugs in history and anthropology, pp. 11-46. Londres: Routledge.

SILVER, Ian A. (1969) «The ageing of domestic animals», en D. Brothwell y E.S. Higgs (Eds.), Science in Archaeology: a survey of progress and research, pp. 283-302. Londres: Thames \& Hudson.

SIMONET, Francisco Javier (1983) Historia de los mozárabes en España. Madrid: Viuda e hijos de M. Tello.
SIMOONS, Frederick J. (1994) Eat not this flesh. Food avoidances from Prehistory to the Present. Wisconsin: The University of Wisconsin Press.

SIMPSON, George G.; ROE, Anne y LEWONTIN, Richard C. (1960) Quantitative zoology. Nueva York: Harcourt, Brace and World.

TURNER, John C. (1999) "Some current issues in research on social identity and self- categorisation theories", en N. Ellemers, R. Spears y B. Doosje (Eds.), Social identity. Context, commitments, content, pp. 6-34. Oxford: Blackwell.

VÁZQUEZ DE PARGA, Luis; LACARRA, José Ma y URÍA RÍU, Juan (1948) Las peregrinaciones a Santiago de Compostela (Tomo I). Madrid: Consejo Superior de Investigaciones Científicas, Escuela de Estudios Medievales.

VIRÉ, François (1986) «Khinzir», Encyclopaedia of Islam, Second Edition, Vol. 5, pp. 8-9. Brill: Leiden.

WAGNER, Heather P. (1991) «Review of the European Pectinidae», Vita Marina, 41, 1-48. 